\title{
Hippocampal Ensemble Activity during Spatial Delayed-Nonmatch-to-Sample Performance in Rats
}

\author{
Sam A. Deadwyler, Terence Bunn, and Robert E. Hampson \\ Department of Physiology and Pharmacology, Neuroscience Program, Bowman Gray School of Medicine, Wake Forest \\ University, Winston-Salem, North Carolina 27157
}

Multiple-cell recording from specially designed arrays of microwire electrodes allowed analysis of anatomically defined ensemble activity from 10 different locations within the hippocampus of rats $(n=7)$ performing a two-lever operant version of a spatial delayed-nonmatch-to-sample task (DNMS). Application of population analysis procedures to ensembles of single-neuron activity within the CA1 and CA3 fields revealed firing patterns related to task-relevant events within a DNMS trial. The patterns were extracted via a canonical discriminant analysis in the form of "roots" that represented sources of variance in firing within the ensemble, such as phase of the task (Sample or Nonmatch), spatial position of the lever press response (left or right), and correct versus error trials. Comparison of the ensemble firing on correct versus error trials revealed important insight into ensemble information encoding, such as "miscoding" of the response position and lack of distinct encoding of the response in the Sample phase, which became increasingly vulnerable to error as a function of the duration of delay interval. The extracted discriminant scores were reflective of multiple representations within ensembles and suggested that "conjunctions" of task-relevant features could be represented effectively by small numbers of hippocampal neurons. The findings support the long-held supposition that hippocampal neurons play a critical role in the encoding and retrieval of information in recognition memory tasks.

Key words: hippocampus; memory; multineuron; electrophysiology; population analysis; behavior; encoding; representation
Performance in delayed-matching-to-sample (DMS) and delayednonmatching-to-sample (DNMS) tasks is one of the few behavioral contexts that can be used to demonstrate hippocampal involvement in recently acquired memories (Gaffan et al., 1984; Rawlins et al., 1988; Weiskrantz, 1991; Gaffan and Murray, 1992; Squire, 1992). There is considerable evidence implicating the hippocampus and, more recently, related structures in the delaydependent accuracy of performance in these tasks (Dunnett, 1985; Markowska et al., 1989; Murray et al., 1989; Zola-Morgan et al., 1989c; Peinado-Manzano, 1990; Tonkiss et al., 1990; Aggleton et al., 1992; Gaffan and Murray, 1992; Otto and Eichenbaum, 1992; Volpe et al., 1992; Angeli et al., 1993; Compton, 1993; Gaffan, 1993; Jarrard, 1993; Rawlins et al., 1993; Suzuki et al., 1993). Studies in animals have attempted to demonstrate functionally related hippocampal cell discharges during the performance of different versions of DMS and DNMS tasks (Miyashita et al., 1992; Young and Yamane, 1992; Miller et al., 1993; Eichenbaum et al., 1994). Results indicate that hippocampal cell activity becomes entrained to different aspects of the task contingency (i.e., behavioral requirements). Hence, cells within the hippocampus and related structures fire not only within the appropriate stimulus contexts but specifically during task-relevant behavioral responses (Deadwyler et al., 1979; Berger et al., 1983; Baylis and

Received June 30, 1995; revised Sept. 25, 1995; accepted Sept. 29, 1995.

This work was supported by NIDA Grants DA03502 and DA00119 to S.A.D. We thank Dr. D. J. Woodward, J. K. Chapin, M. Nicolelis, and L. J. Porrino for helpful comments. We thank V. C. King, M. T. Kirby, D. R. Byrd, and J. K. Konstantopoulos for technical support.

Correspondence should be addressed to S. A. Deadwyler, Department of Physiology and Pharmacology, Bowman Gray School of Medicine, Medical Center Blvd., Winston-Salem, NC 27157-1083.

Copyright (C) 1995 Society for Neuroscience $0270-6474 / 95 / 160354-19 \$ 05.00 / 0$
Rolls, 1987; Miller, 1991; Miller et al., 1991; Hampson et al., 1993b).

It now appears that the hippocampus, defined narrowly to include only the various subfields excluding retrohippocampal areas such as perirhinal, subicular, and parasubicular regions, plays a more restricted role relative to other structures in the performance of tasks that involve DNMS requirements (Eichenbaum et al., 1994; Alvarez et al., 1995). There is some evidence, however, that the well known spatial mapping function of hippocampal cells (O'Keefe and Nadel, 1978; Nadel, 1991) can be modulated by other task demands as revealed by encoding of reinforcement-contingent behavioral events by hippocampal neurons (Eichenbaum et al., 1987; Breese et al., 1989; Wiener et al., 1989; Hampson et al., 1993a). Although the role of hippocampal unit activity in DNMS-tested memory in rats and primates certainly has not been defined (Funahashi et al., 1989; Sakurai, 1990a,b; Miyashita et al., 1992; Otto and Eichenbaum, 1992; Rolls et al., 1993; Eichenbaum et al., 1994; Gluck and Myers, 1995), it is apparent that the functional significance of hippocampal cellular activity measured electrophysiologically in animals engaged in these tasks must conform to the degree to which performance is affected by hippocampal damage or removal. Given that hippocampal cell ensembles represent information critical to DNMS performance and that an intact hippocampus provides the capacity to perform this task at maximum efficiency, it is necessary to demonstrate (1) that hippocampal cells exhibit a representational code for critical DNMS task-relevant events and (2) that when this representation is not present, is distorted, or is diminished, there is a corresponding decrement in DNMS performance. A third requirement is that this process be susceptible to decay or perturbation in a delay-dependent manner corresponding to the nature of the behavioral deficit as expressed by DNMS performance curves. 


\section{A. DNMS TASK}

RAMPLE DELAY NONMATCH

\section{B. DNMS PERFORMANCE}

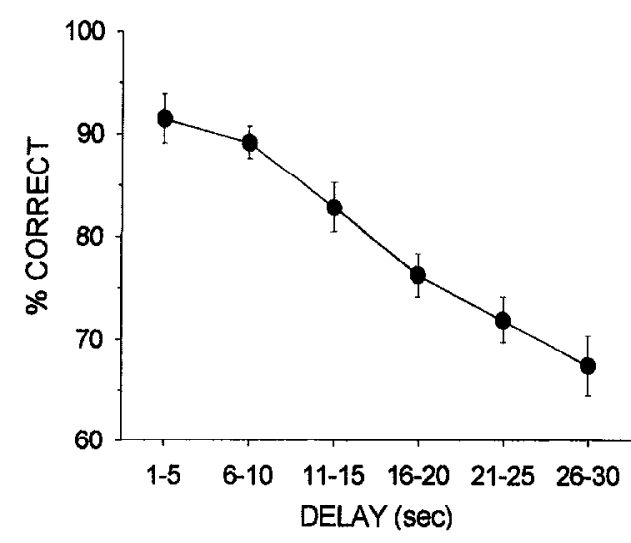

Figure 1. A, Schematic of Sample, Delay, and Nonmatch phases of the spatial DNMS task. A left Sample trial is shown. Lever is presented (left lever present) during the Sample phase of the task and is retracted after the lever press (left press). Nosepoke responses (bottom) are required to terminate the interposed delay phase. Both levers are presented (left and right present) during the Nonmatch ("decision") phase of the task; a response on the right lever (right press) satisfies the Nonmatch contingency and is reinforced with a water reward $\left({ }^{*}\right)$. The dotted "blip" (arrow) denotes an error (i.e., "match") response on the same type of trial. $B$, Delay-dependent performance in the DNMS task. Mean ( \pm SEM) percent correct trials per session (100-200 trials) was averaged across all seven animals (5-10 sessions each) in which electrophysiological measures were taken. Trials were grouped and plotted by $5 \mathrm{sec}$ intervals across the $1-30 \mathrm{sec}$ delay periods.
In the present study, ensembles of 10 neurons were recorded from electrode arrays strategically placed in the CA1 and CA3 fields of the hippocampus in seven different rats performing at criterion on a two-lever spatial DNMS task at delays of 1-30 sec. Population statistical procedures consisting of canonical discriminant analyses (Lawley, 1959; Stevens, 1992; Nicolelis and Chapin, 1994) were used to assess patterned activity (Young and Yamane, 1992; Miller et al., 1993; Nicolelis et al., 1993a,b) within the hippocampal ensembles. Two different error-generating processes, one of which was delay-dependent, were identified by comparison with patterns of ensemble activity on correct trials. The findings reveal that correct performance in the DNMS task required successful encoding of different task-related events within the ensemble and provide considerable insight into the nature of representational systems engaged during performance of recognition memory tasks.

\section{MATERIALS AND METHODS}

Spatial DNMS task. Male Sprague-Dawley rats, 280-300 gm $(n=7)$, were water-deprived for $22 \mathrm{hr}$ and trained to stable DNMS performance levels on a modified version of the Dunnett two-lever spatial DMS task (Dunnett, 1985, 1989; Dunnett et al., 1989) with variable delay intervals 1-30 sec in duration (Hampson et al., 1993b; Heyser et al., 1993). The delay intervals varied randomly with a resolution of $1.0 \mathrm{sec}$ within the session. Figurc $1 A$ diagrams the bchavioral cvents of a DNMS trial. At the start of each trial, one of two levers, positioned on either the left or right side of the front panel of the chamber, was extended, thereby initiating the Sample phase of the task. A lever press response on the extended lever initiated the delay period and retracted the lever. During the delay period, the animal was required to nosepoke in a device illuminated by an overhead cue light, located on the opposite wall of the chamber. A nosepoke that occurred after the delay interval timed out, extinguished the cue light and terminated the delay period, initiating the Nonmatch phase of the task. The Nonmatch phase was characterized by extension of both levers into the chamber. A response on the lever opposite the one pressed in the Sample phase (i.e., a "Nonmatch" response) was rewarded with a drop of water delivered to a trough located between the two levers. An intertrial interval of $10 \mathrm{sec}$ was interposed between the termination of a trial and presentation of the Sample lever for the next trial. When an error occurred (i.e., a "match" response), the overhead lights in the box were extinguished immediately for $5 \mathrm{sec}$ and no water was delivered. A new trial was initiated (Sample lever extended) 5 sec later. Each session contained 100-200 DNMS trials.

All animals $(n=7)$ were well trained $(30-60 \mathrm{~d})$ and performed the DNMS task at criterion levels ( $\geq 90 \%$ correct at $1-5$ sec delay) during all sessions in which electrophysiological data were collected. Figure $1 B$ shows the mean performance curve for all seven animals averaged over 5-10 sessions for each animal. Delay-dependent performance was exhibited by each animal (Dunnett, 1985; Hampson et al., 1993b; Heyser et al., 1993 ) for delays of $1-30 \mathrm{sec}$ (decrease from 95 to $65 \%$ correct was significant, $F_{(5,30)} \geq 8.70, p<0.01$ ). At least 700 single trials were recorded per animal across a range of 5-10 DNMS sessions. Differences with respect to response latencies, number of trials to criterion, number of error trials, etc., were examined and shown not to affect the neuron activity described below. Perhaps most important, there were no significant differences in performance of the task between animals $\left(F_{(6,239)}=1.32, p>0.10\right)$.

Surgical procedure. Each animal was anesthetized with ketamine (100 $\mathrm{mg} / \mathrm{kg})$ and xylazine $(10 \mathrm{mg} / \mathrm{kg})$ and stereotaxically implanted with a specially designed multiple-microwire $(50 \mu \mathrm{m})$ electrode array. The array was positioned at the time of surgery with the tips of the electrodes above or within the cell layers of the CA1 and CA 3 subfields of the hippocampus (Fig. 2A). The center pair of array electrodes was positioned at coordinates $3.8 \mathrm{~mm}$ posterior to bregma, $3.0 \mathrm{~mm}$ left of midline. The array was driven in $25 \mu \mathrm{m}$ steps to a depth of $3.0-4.0 \mathrm{~mm}$ for CA3 leads, with the CA1 leads automatically positioned $1.2-1.4 \mathrm{~mm}$ higher. Neural activity from the microwire electrodes was monitored throughout surgery (Hampson et al., 1993b; Heyser et al., 1993). After array placement, the cranium was sealed with bone wax and dental cement and the animal was allowed to recover. The scalp wound was treated periodically with Neosporin antibiotic, and animals were given an injection of Crysticillin (penicillin $\mathrm{G}, 300,000 \mathrm{U}$ ) to prevent infection. All animal care and experimental procedures conformed to National Institutes of Health and Suciely for Neuroscience guidelines for care and use of experimental animals.

Multineuron recording technique. Neural activity (extracellular action potentials, "spikes") and behavioral responses were digitized and timestamped for computer processing in relation to successive behavioral "events" within each DNMS trial as shown in the stripchart display in Figure $2 B$. Ten neurons, one from each wire, were isolated and selected for analysis from the 16 different locations on the recording array (Hampson et al., 1992, 1993b, 1994). Neuronal action potentials were digitized at $40 \mathrm{kHz}$ and isolated by time-amplitude window discrimination as well as computer-identified individual waveform characteristics. Identified spikes from selected wires were "tracked" from session to session by waveform and firing characteristics within the task (perievent histograms). It is possible that the neuronal spikes discriminated on a given wire did not consistently identify the same neuron (McNaughton et al., 1983). However, only spike waveforms with associated firing rates and perievent histograms (i.e., behavioral correlates) that were consistently the same across sessions were included in the analysis to maximize the likelihood that the same neurons were recorded continuously.

Population statistical analyses of ensemble activity. Canonical discriminant analyses (PROC CANDISC, SAS, Cary, NC) (Lawley, 1959; Stevens, 1992) were carried out on multiple-neuronal spike activity (ex(racellular action potentials) to detect and classify recurring patterns of activity within the ensemble (Nicolelis and Chapin, 1994). The design of the analysis is shown in Figure $3 A$, in which three categories-trial phase (Sample and Nonmatch), trial type (correct or error), and lever position (left or right)-accounted for eight distinct behavioral event classifications. An additional element, length of delay, was also analyzed separately with respect to features extracted via the canonical discriminant analysis. The data were sorted into two categories consisting of all trials with delays $<15 \mathrm{sec}$ and all trials with delays $\geq 15 \mathrm{sec}$. The neural data obtained from each animal consisted of spikes from five isolated CA1 
A.

\section{ELECTRODE ARRAY}

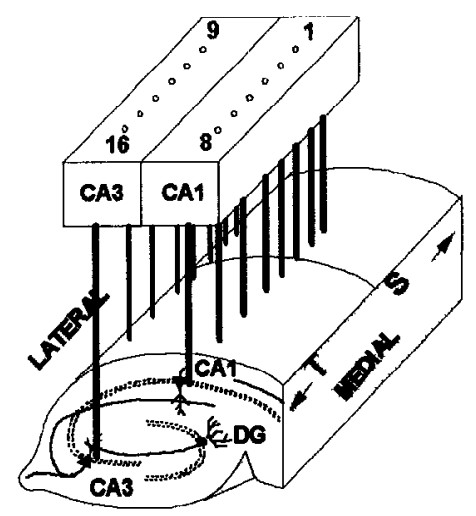

B.

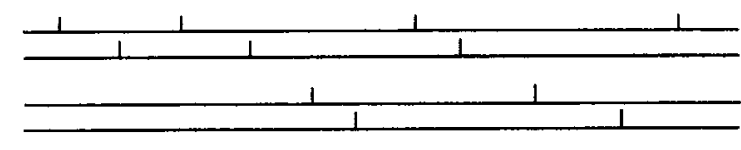

LSAMPLE R-NONMATCH R-SAMPLE L-NONMATCH NOSEPOKE
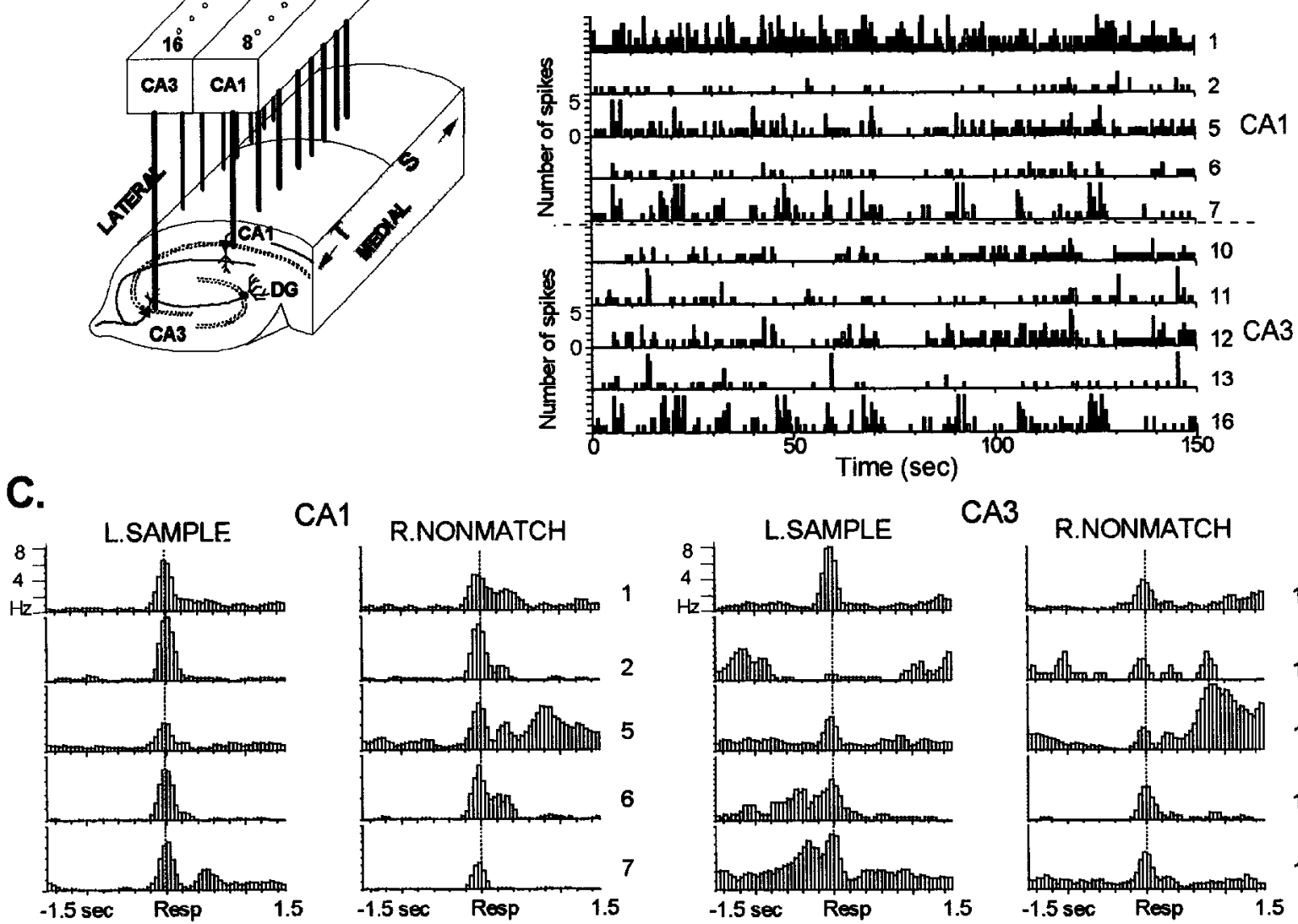

CA3

Figure 2. A, Diagram of microwire electrode recording array, consisting of two parallel rows (row separation $0.8 \mathrm{~mm}$ ) of $50 \mu \mathrm{m}$ stainless steel microwires forming eight pairs (pair separation $200 \mu \mathrm{m}$ ) of electrodes along the septo-temporal $(S$ and $T$ ) axis of the hippocampus. The lateral row of electrodes was positioned to record from the CA3 subfield of the hippocampus, whereas the medial row was positioned in the CA1 subfield. $B$, Stripchart of behavioral events and ensemble activity recorded continuously over five DNMS trials $(150 \mathrm{sec})$ for a single animal. Single neurons recorded from individual microwires on the array shown in $A$ were isolated and identified by time-amplitude window discrimination of extracellular action potential waveforms. Extracellular spike events were sampled from only one neuron at each of 10 different array locations and stored along with behavioral events in time-stamped computer files. Neural activity (spike firing) is plotted as the number of spikes (vertical scale) recorded per $500 \mathrm{msec}$ time bin. Lever press responses and nosepokes (compare Fig. 1A) are indicated by the single "tick" marks as indicated. Individual neurons identified by numbers at the right refer to the corresponding locations on the recording array shown in $A . C$, Perievent histograms summed over all L.Sample-R.Nonmatch trials in the same session as in $B$. Spike events $1.5 \mathrm{sec}$ before and after each behavioral response were summed in $50 \mathrm{msec}$ bins and normalized by number of trials to show mean firing rate (in $\mathrm{Hz}$ ) for each neuron in the ensemble. Because each trial consisted of two responses, one in the Sample and one in the Nonmatch phase, example perievent histograms for a single trial (Sample and Nonmatch) are shown. Electrode position on the recording array $(A)$ is indicated at the right for $\mathrm{CA} 1$ and $\mathrm{CA} 3$ neurons, respectively.

neurons and five CA3 neurons making up each ensemble. Each neuron included in the analysis was located on a different wire and had firing characteristics similar to that previously reported for hippocampal complex spike cells, and all theta cells were excluded (Christian and Deadwyler, 1986).

Each DNMS trial was characterized and classified according to the two (out of 4 possible) behavioral responses that occurred within the trial (Fig. $3 A$, top). Analysis of ensemble activity examined firing rates in all 10 neurons $1.5 \mathrm{sec}$ before and after each behavioral event (response) within a trial. The canonical discriminant analysis, as applied in the current study, detected patterns of covariance in firing rates between neurons in the ensemble and determined which combinations of neurons were most effective for discriminating these patterns. The analysis extracted discriminant functions, or "roots," that defined the relationship between firing rate within the ensemble $\left(X_{n, t}\right)$ and different patterns of covariance characterized by the derived coefficients $\left(W_{n, t}\right)$ within the same ensemble. The discriminant functions used linear equations of the form $\left(X_{n, t} \cdot W_{n, t}\right)$ to weight each neuron's firing rate according to its "contribution" to a particular pattern of covariance extracted by that root. The resulting patterns of weighted, or "adjusted," firing rates for each trial then could be either displayed and analyzed for the entire ensemble or summed across neurons to provide a single "discriminant score" reflective of the contribution of a given root to overall ensemble activity. Ensemble activity, therefore, could be described by multiple discriminant functions, each of which reflected a different variance source within the neuron population.

Procedures for obtaining canonical discriminant roots are depicted schematically in Figure 3. Single-trial ensemble firing for each event formed a two-dimensional data vector $\left(X_{n, t}\right)$ corresponding to number of spikes $(X)$ from each neuron $(1,2, \ldots, n)$ in each time bin $(1,2, \ldots, t)$ over the $3.0 \mathrm{sec}$ response analysis epoch. Cross-covariance matrices $\left(A_{n, t}\right.$ etc., Fig. $3 A$, middle) were then constructed from the data vectors for the eight different behavioral event classifications. Seven eigenvectors (i.e., one less than the number of event classifications) were derived from the cross-covariance matrices and provided the coefficients (Fig. $3 A, W_{n, t}$ ) of the canonical discriminant functions, or "roots," that identified significant 
A.
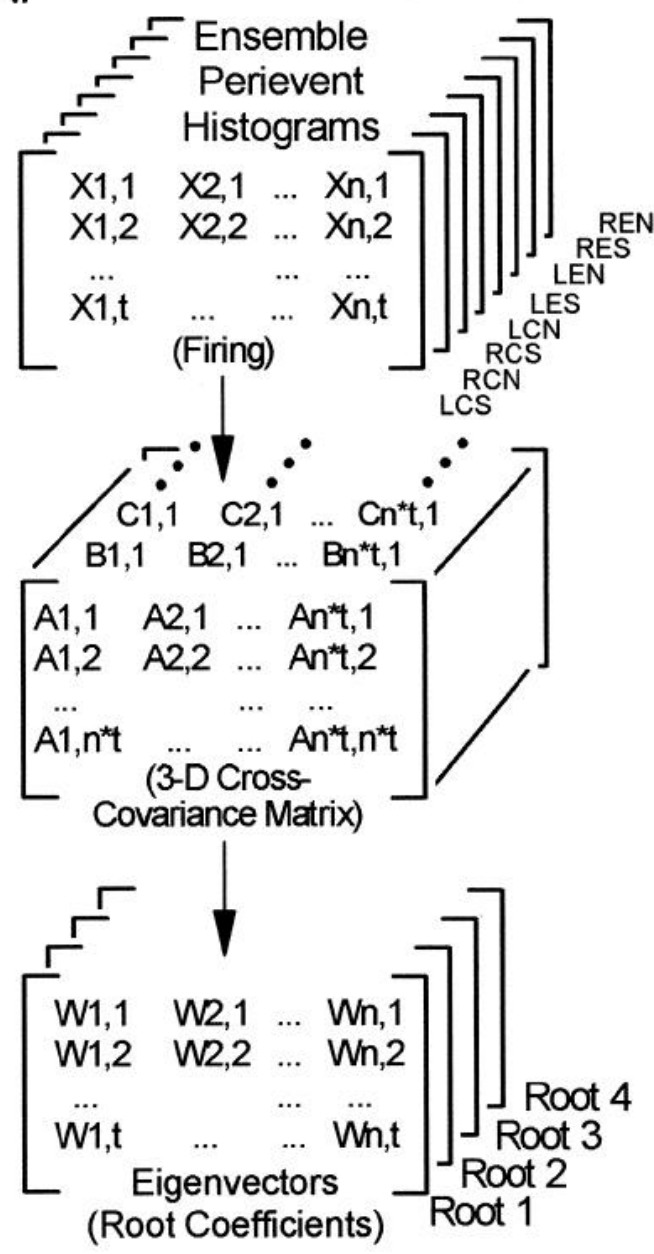

B. Calculation of Single Event Root Discriminant Scores

Discriminant Scores (d.s.) d.s. $=\mathrm{X}_{1,1} * \mathrm{~W}_{1,1}+\mathrm{X}_{1,2} * \mathrm{~W}_{1,2}+\ldots+\mathrm{X}_{\mathrm{n}, \mathrm{t}} * \mathrm{~W}_{\mathrm{n}, \mathrm{t}}$

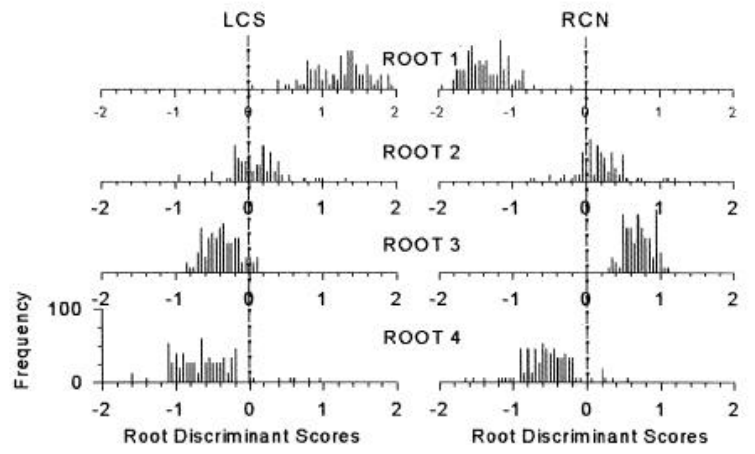

C. Root Adjusted Firing Surface

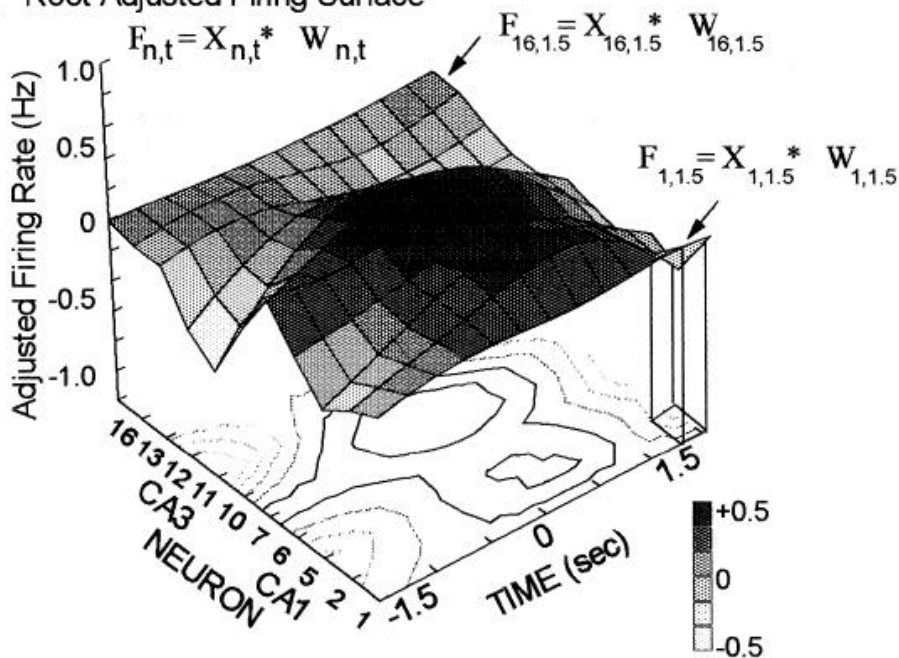

Figure 3. A, Schematic of the canonical discriminant analysis: behavioral responses (events) were categorized according to task phase (Sample or Nonmatch), lever position (Left or Right), and trial performance (Correct or Error), providing eight different classifications (LCS, Left-Correct-Sample; $R C N$, Right-Correct-Nonmatch; RCS, Right-Correct-Sample; $L C N$, Left-Correct-Nonmatch; $L E S$, Left-Error-Sample; $L E N$, Left-Error-Nonmatch; RES, Right-Error-Sample; $R E N$, Right-Error-Nonmatch). Data vectors $\left(X_{n, t}\right)$ were constructed from 120 quantitative variables [10 neurons $\times 12(250$ msec) time bins.] 3-D cross-covariance matrices incorporating firing across neurons, time, and event classifications $(A, B, C$, etc.) were computed from single trials and seven eigenvectors were derived from the cross-covariance matrices. Each eigenvector provided one independent canonical discriminant function $\left(W_{n, t}\right)$ or "root," each accounting for successively less variance in ensemble activity. $B$, Calculation of single-event discriminant scores: coefficients of the discriminant functions $\left(W_{n, t}\right)$ for a given root were used to determine the weighting of each neuron's firing rate within the ensemble. The rate $\times$ coefficient products $\left(X_{n, t} \cdot W_{n, t}\right)$ for each root were normalized (see text) to provide adjusted firing rates for all neurons in the ensemble, then summed to yield single-event discriminant scores. The histograms depict the frequency distribution of discriminant scores within two different behavioral event classifications ( $L C S$ and $R C N$, see $A$ ) for each of the different extracted roots. $C$, Adjusted firing surface across the entire ensemble for a given root: the firing surface was calculated using mean ensemble firing rate across all trials containing a given behavioral event $(n>100$ trials) for a single animal. Location on the recording array (CA1 vs CA3), time $( \pm 1.5 \mathrm{sec})$ before and after the response $(0 \mathrm{sec})$, and adjusted firing rate $\left(F_{n, t}\right)$ of each neuron at each time bin form the three dimensions of the adjusted firing surface. Adjusted rates for two different neurons (1 and 16) at a single time bin (1.5 sec) are indicated by arrows. Adjusted firing rates were computed from the product of the mean neuron spike frequency and root coefficients (i.e., $F_{1,1}=$ $X_{1,1} \cdot W_{1,1}$, etc.). Increases and decreases in adjusted firing rate (in $\mathrm{Hz}$ ) are represented by deflections and shading of the surface. Light-to-dark 2 -D contour lines at the bottom of the graph reflect changes in the overhanging 3-D surface. The surface gray scale range is from $<-0.5$ to $>+0.5 \mathrm{~Hz}$ (white to black). Zero indicates no consistent change in the rate of firing of any of the neurons within the ensemble with respect to the root influences on a particular behavioral event. Positive and negative deflections indicate changes in neuronal firing rates within each corresponding spatiotemporal domains of ensemble activity. Surfaces were smoothed and contoured by a spline interpolation program (CSS; Statistica, StatSoft, Tulsa, OK).

sources of variance in ensemble activity for each of the eight different behavioral events (Stevens, 1992). The identified variance sources (ranked from largest to smallest) were independent (but not necessarily orthogonal) and, hence, could be added or subtracted from the total variance to determine specific contributions. The weighting by the root discriminant function, of each neuron's firing rate within the ensemble, was determined by normalizing and "adjusting" firing rates for each neuron at each $250 \mathrm{msec}$ time bin $\left(X_{1,1} \cdot W_{1,1}, X_{1,2} \cdot W_{1,2}, \ldots X_{2,1} \cdot W_{2,1}\right.$, $\left.\ldots X_{n, t} \cdot W_{n, t}\right)$ in the $3.0 \mathrm{sec}$ analysis epoch. The adjusted firing rates could also be summed into a single discriminant score (i.e., $X_{1,1} \cdot W_{1,1}+$
$\left.X_{1,2} \cdot W_{1,2}+\ldots+X_{2,1} \cdot W_{2,1}+\ldots+X_{n, t} \cdot W_{n, t}\right)$ for each root, and for each behavioral event. These discriminant scores thus provided a single value (score) that represented each root's relative contribution to ensemble firing for a given event in a given single trial. The scores for any one root were distributed normally with mean $=0$ and $\mathrm{SD}=1$, such that a difference in mean scores of \pm 0.75 was statistically significant $(p<0.01)$.

Identification of the sources of variance with respect to DNMS behavior was determined by examining the single-event discriminant scores to determine whether they were consistently dispersed along the dimension of classified behavioral events (Fig. $3 B$ ). If the scores proved to be 


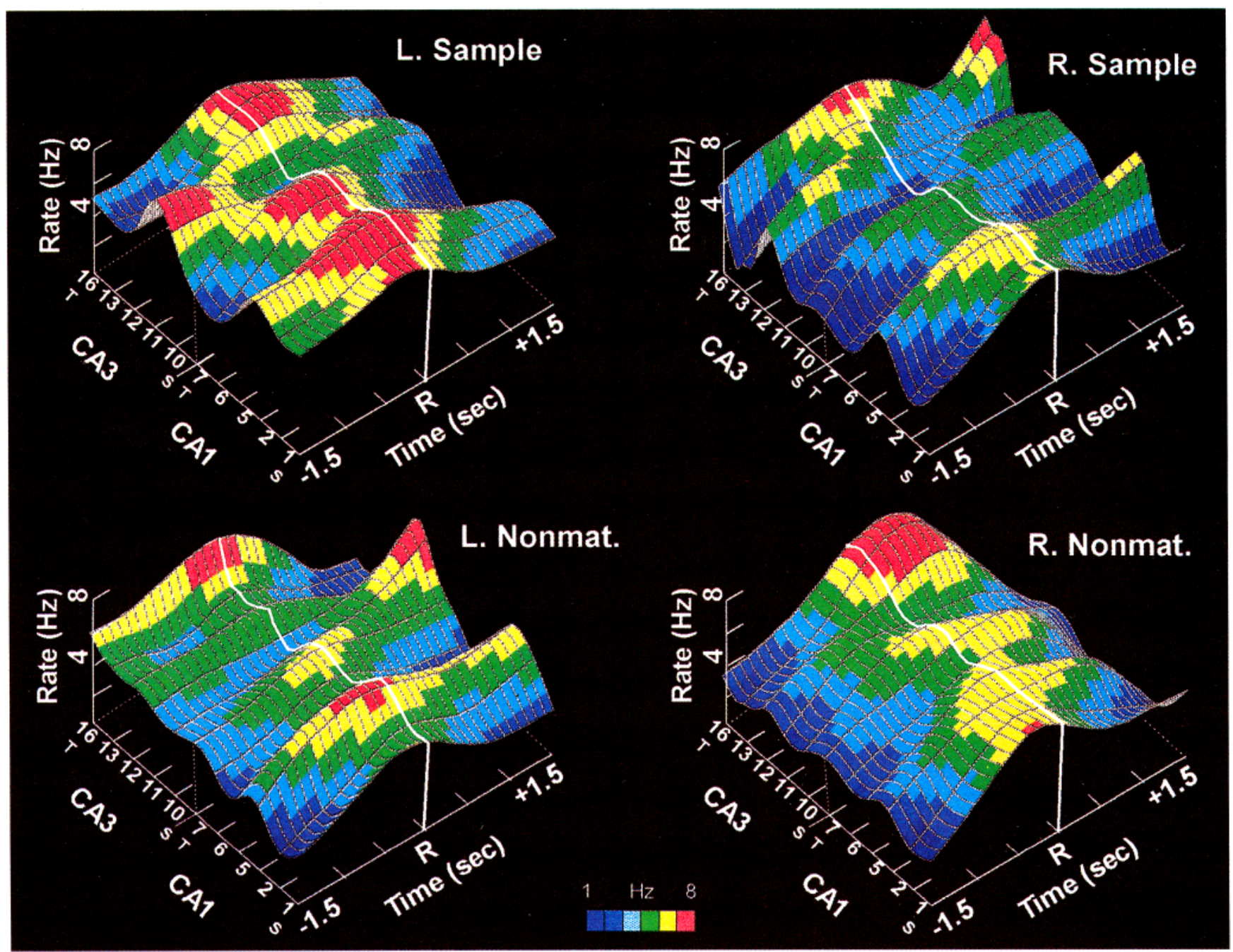

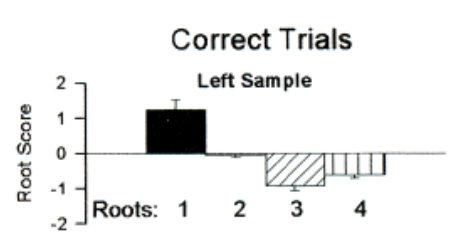
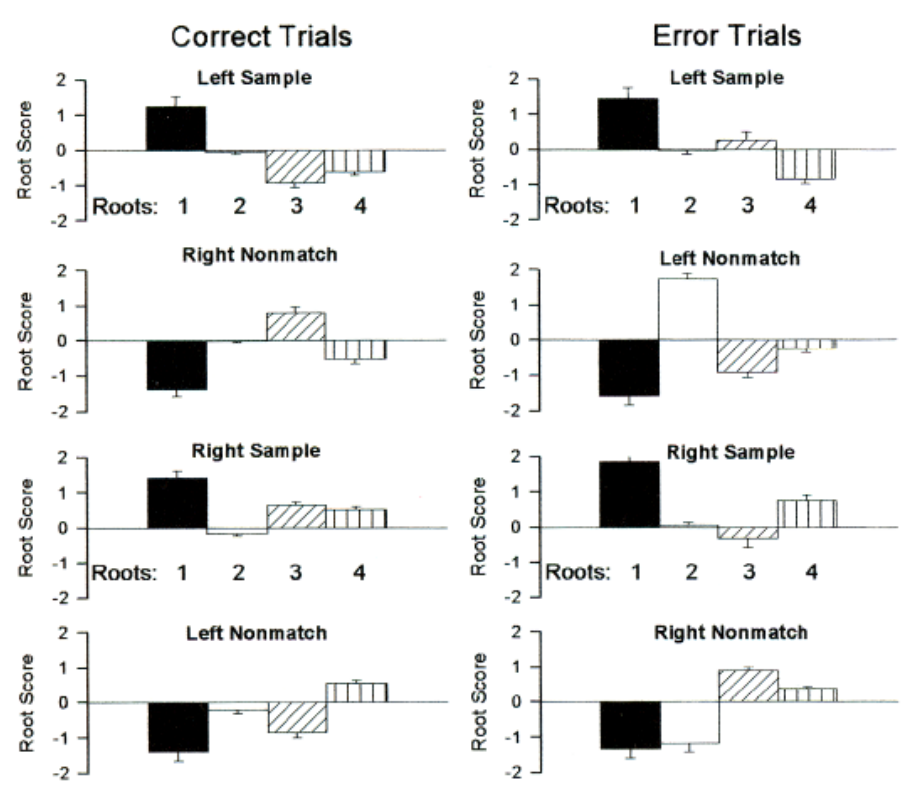

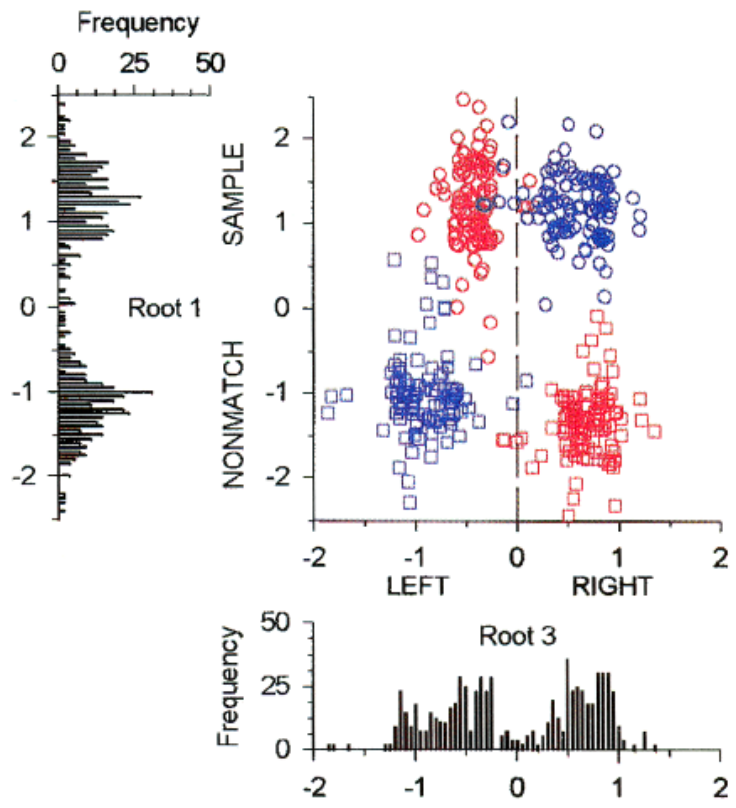


distributed with respect to a dimension of DNMS task relevance (e.g., position of lever press), then that source of variance or "root" was assumed to be generated by features and contingencies operating within the DNMS task. If not, then the extracted roots might reflect factors not explicitly controlled by the DNMS contingencies, but of sufficient magnitude to systematically affect the ensemble firing pattern (e.g., theta rhythm whenever the animal moved). Figure $3 B$ shows the frequency histograms of discriminant scores from Roots 1-4 for two different behavioral events within a trial (LCS and RCN). The categories of event classification (i.e., task phase, lever position, and performance) discriminated by each root are indicated by the groupings of discriminant scores along the horizontal axis in Figure $3 B$. Changes within a category isolated by each root are indicated by a shift in the location of scores as a function of different behavioral events. In all instances in the present study, the sources of variance extracted by the analysis, which were statistically significant, were associated with dimensions of responding directly related to DNMS performance accuracy. In addition, the extracted roots were consistent across all seven animals with respect to identified sources of variance and ranked by percent variance contribution.

The influence of each of the extracted roots on the ensemble firing pattern was demonstrated independently by constructing three-dimensional (3-D) firing surfaces for individual roots by plotting the adjusted firing rates (i.e., $X_{n, t} \cdot W_{n, t}$ Fig. $3 C$ ) of each of the 10 neurons within the ensemble as combined perievent histograms. Each adjusted firing surface was constructed from a single animal's data and for each behavioral event and illustrated the spatiotemporal distribution of firing within the ensemble exemplified by each root (Fig. $3 C$ ). The adjusted firing surface shown in Figure $3 C$ was constructed from the perievent histograms shown in Figure $2 C$, and the coefficients for Root 3 shown in Figure $3 B$ (LCSevent). On the firing surface, adjusted rates ranged from -0.5 to $+0.5 \mathrm{~Hz}$ as shown by the gray scale in Figure $3 C$ (rates were plotted within \pm 1.0 to avoid distortion). Thus, for each significant source of variance in neural activity (rool) within an ensemble, a representative "adjusted" firing surface could be constructed specifically for a given behavioral event. Because the root coefficients extracted a pattern of firing from the covariance matrix that was derived from all behavioral events, comparison between adjusted 3-D firing surfaces illustrates differences in ensemble firing associated with different task-relevant behaviors. The root coefficients, therefore, act as "masks" through which the spatiotemporal distribution of neural activity associated with a particular dimension (phase, position, performance) of DNMS behavior can be identified (Fig. 3A).

\section{RESULTS}

\section{Spatiotemporal distribution of neuron firing within ensembles}

Hippocampal ensemble activity was recorded from seven different animals during criterion performance of the DNMS task. Identi- fied recordings from the same hippocampal locations across multiple sessions in each animal provided the data for examination of ensemble firing characteristics. Figure 4 shows the distribution of (unadjusted) hippocampal neuronal firing in the ensemble as 3-D firing surfaces in relation to both time and neuron location in the hippocampus (as demarcated by the electrode array in Fig. $2 A$ ). The spatiotemporal patterns corresponding to the four different behavioral events making up successful DNMS performance on correct trials are shown in Figure 4 as diagonal 3-D firing surfaces (i.e., L.Sample-R.Nonmatch). It is quite clear that there was more similarity in ensemble firing surfaces within correct DNMS trials (diagonal surfaces) than between similar behavioral events not related to successful DNMS performance (i.e., L.Sample surface vs L.Nonmatch surface). The illustrated Sample and Nonmatch phase firing patterns "dissolved" and were not present in ensemble activity during the delay period or intertrial interval, respectively, a finding reported previously for single hippocampal neurons in DMS tasks (Hampson et al., 1993; Heyser el al., 1993). Figure 4 shows that firing patterns similar to those in the Sample phase reappeared, however, in the same temporal relation to the response in the Nonmatch phase of the task. Overall ensemble firing rates for the Sample (mean $=4.51 \pm$ $0.29 \mathrm{~Hz}$ ) and Nonmatch (mean $=5.22 \pm 0.35 \mathrm{~Hz}$ ) phases of the task were 3-5 times higher than firing rates within the delay period $($ mean $=1.56 \pm 0.11 \mathrm{~Hz})$.

Close inspection of the firing surfaces showed a large degree of variability in patterns of activation across bchavioral events. This suggests that neural activity associated with simple motor or sensory components involved in movements toward the levers and execution of responses was not a major contributor to firing depicted on the surfaces (i.e., differences between L.Sample and L.Nonmatch firing surfaces), because these movements were well trained, very stereotyped, and consistent for all behavioral events. In addition, detailed examination of differences in patterns of traversal between the levers during the two phases of the task also did not reveal consistent differences due to locomotor activity that could account for the correlation of ensemble neural activity with DNMS event classification. However, similar features could be observed in the firing surfaces for different events within animals. For example, in the surfaces depicted in Figure 4, the alternating

$\leftarrow$

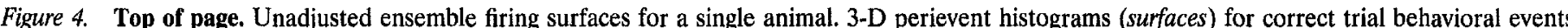

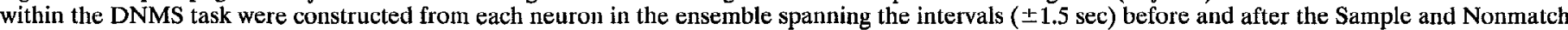

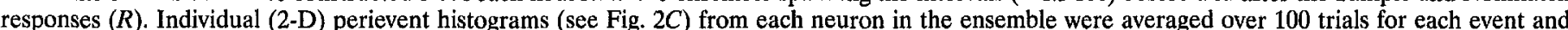

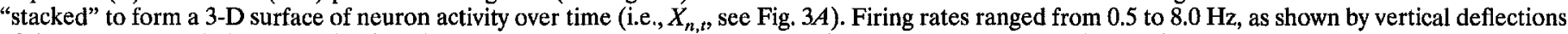

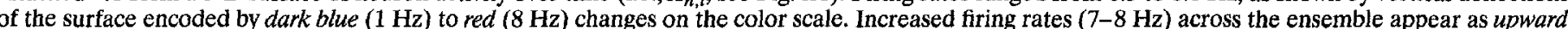

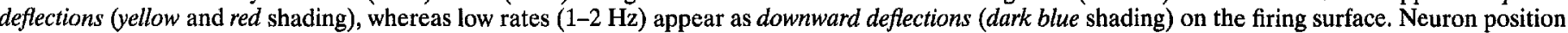

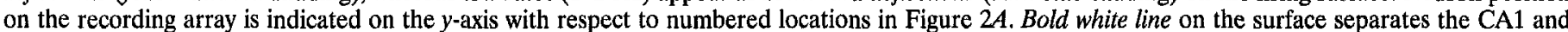

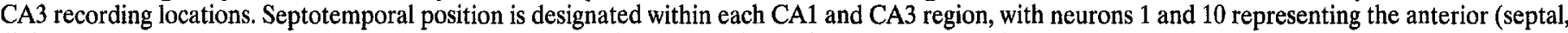
$S$ ) locations, and neurons 7 and 16 representing posterior (temporal, $T$ ) locations on the recording array.

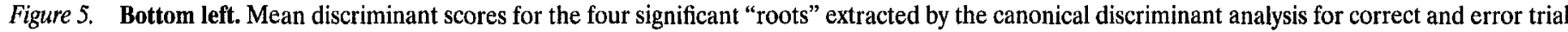

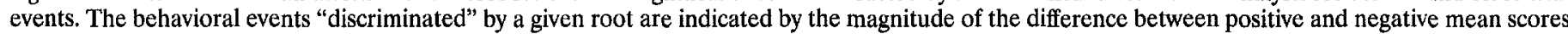

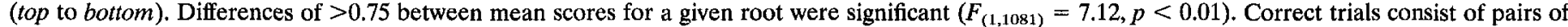
L.Sample-R. Nonmatch or R.Sample-L.Nonmatch events. Error trials consist of L.Sample-L.Nonmatch, or R.Sample-R.Nonmatch events.

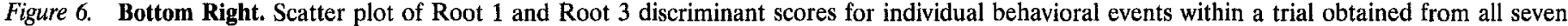

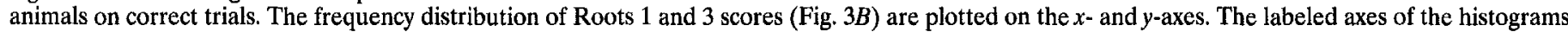

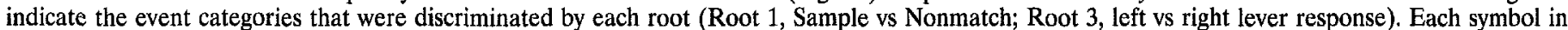

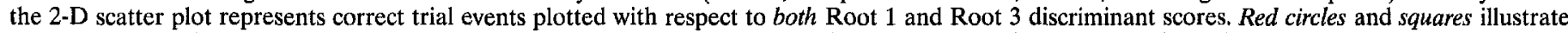

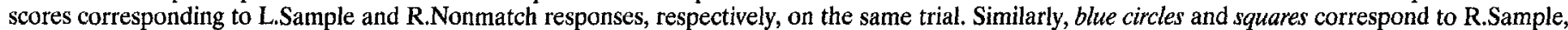

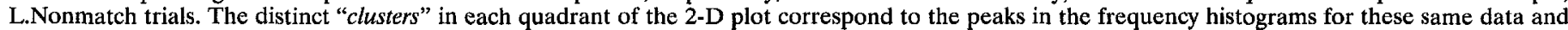

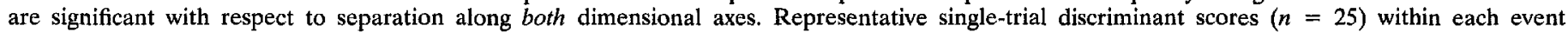
(quadrant) were randomly selected and plotted from each of the seven different animals (175 scores/cluster, $\sim 10 \%$ of total trials). 
regions of high (peaks) and low (valleys) neural activity indicated that adjacent neurons in both the CA1 and CA3 locations of the electrode array were either active or inactive in a time-locked manner to the behavioral event. There was also a definite tendency for some neurons to be more active than others as indicated by the "wave-like ripples" apparent in the firing surface in Figure 4.

Another general feature illustrated in Figure 4 was that some neurons in the ensemble participated in the generation of both patterns within a trial (large "mound" in CA3 after the response in L.Sample and R.Nonmatch surfaces), whereas others were activated during only one type of behavioral event (differentially located "sharp peaks" at $+1.5 \mathrm{sec}$ on the R.SampleL.Nonmatch surfaces in Fig. 4). Regardless of whether the differences in ensemble firing surfaces were generated by the same neurons or possibly by more than one neuron inadvertently recorded from the same location (McNaughton et al., 1983), the consistency with which the particular patterns recurred across trials and sessions in the same animals indicates that the spike identification techniques used (see Materials and Methods) were sufficient to detect behavioral selectivity in ensemble firing. This was verified in a much more detailed manner by the results of the canonical discriminant analysis that necessarily identified changes in ensemble firing patterns by extracting specific sources of variation at each neuron location that were consistently present on a trial-to-trial basis (see below).

\section{Canonical roots related to DNMS task-specific ensemble activity}

Figure 5 displays the overall results of the canonical discriminant analysis in terms of the mean discriminant scores for the four significant "roots" extracted in each of the seven animals. These four canonical roots contributed a mean of $87.5 \%$ of the ovcrall variance in normalized discriminant scores of ensemble activity across the eight different DNMS behavioral event classifications shown in Figure $3 A$. The remaining three roots together contributed $<13 \%$ of the overall variance in ensemble activity, and none of these reached statistical significance. Figure 5 illustrates the relationship of the four significant "roots" to the individual behavioral events in the DNMS task. The discriminant scores for each of the four roots across all ensembles were computed, then averaged to produce a single mean score across all animals. The four roots ranked similarly in terms of percent variance contribution and behavioral events discriminated in each of the seven animals, indicating that the same types of influences on firing within a DNMS trial were present in all animals.

\section{Root 1: Sample versus Nonmatch phase}

Root 1 accounted for the largest significant percentage of overall variance in each of the seven different animals (mean $=56.3 \% \pm$ $\left.4.2 \mathrm{SEM}, F_{(120,1081)}=14.8, p<0.001\right)$. There were two distinct groups of discriminant scores for Root 1 , one specific to the Sample phase in which the mean was $1.54 \pm 0.24$ SEM and another for the Nonmatch phase in which the mean score was $-1.68 \pm 0.25$ SEM (Fig. 5). Figure 5 shows the mean discriminant scores for Root 1 for each of the eight behavioral events. The distinctly different firing patterns represented by Root 1 for the Sample and Nonmatch phases of the trial depend neither on the position (left or right) of the lever press response in either phase nor whether it was a correct or an error trial (Fig. 6). This result indicates that the canonical discriminant analysis extracted changes in the firing of neurons within each of the ensembles that differentiated specifically between the Sample and Nonmatch phases of the task.

\section{Root 2: Nonmatch phase errors}

The next significant canonical root, Root 2, contributed a mean of $11.7 \% \pm 2.1 \operatorname{SEM}\left(F_{(120,1081)}=4.38, p<0.001\right)$ of the overall variance in normalized discriminant scores (Fig. 5). The source of variance represented by Root 2 was related to commission of an error in the Nonmatch phase of the task. Figure 5 (error trials) shows that Root 2 scores differentiated between left and right lever responses during the Nonmatch phase of the task on error trials only. The absence of significant Root 2 mean scores for all other events demonstrates that it did not distinguish firing during correct trials or in the Sample phase on error trials (Fig. 5). Thus, variance associated with Root 2 was exclusive to behavioral errors committed within the Nonmatch phase of the task (left- and rightNonmatch). The significance of this source of variability in ensemble activity will be discussed below in connection with error processes operating on DNMS trials.

\section{Root 3: lever position}

The source of variance extracted by Root 3 in the analysis was identified with discrimination of spatial position of the levers. Figure 5 indicates that the mean discriminant scores of all ensembles varied distinctly as a function of position of the lever press response, regardless of the phase of the task or whether the trial was correct or an error. Root 3 accounted for a mean of $10.3 \% \pm$ $0.8 \mathrm{SEM}$ of the overall variance in ensemble activity and represented a statistically significant $\left(F_{(120,1081)}=3.40, p<0.001\right)$ source of variance related to lever position. As with Roots 1 and 2 , there were two groups of scores generated by Root 3 in the overall analysis (Fig. 3), one associated exclusively with right lever presses $($ mean $=+0.58 \pm 0.21 \mathrm{SEM})$ and the other with left lever presses (mean $=-0.64 \pm 0.18 \mathrm{SEM}$ ). In Figure 5 , the alternating positive and negative values of the scores reflect the fact that Root 3 varied within each trial because of the nonmatch response contingency. There was also a systematic difference in Root 3 scores during Sample responses on error trials (Fig. 5) compared with correct trials that will be discussed below. Roots 1 and 3 , therefore, reflected major sources of task-relevant "information" within the DNMS trial.

\section{Root 4: Sample "position reference"}

The last significant source of variance extracted in all ensembles by the canonical analyses was Root 4 , which accounted for a mean of $9.2 \% \pm 1.0 \mathrm{SEM}$ of the overall variance $\left(F_{(120.1081)}=2.50\right.$, $p<0.001$ ) in ensemble firing (Fig. 5). Two distinct groups of discriminant scores were extracted by Root 4 which, like Root 3 , varied along a dimension exclusively related to lever position. However, the position represented by Root 4, even in the Nonmatch phase, was always associated with the position of Sample phase response. In other words, Root 4 mean scores were the same in the Nonmatch phase as in the Sample phase even though the actual behavioral response in the Nonmatch phase occurred at the opposite lever position. On correct and error trials, Root 4 discriminant scores in both phases of the task were not significantly different. 'Thus, Root 4 discriminated the position of response in the Sample phase of the task likc Root 3 but, unlikc Root 3, "rctaincd" that Samplc phase score in the Nonmatch phase at the time of the response. The consistent reappearance of Root 4 scores with the same values as in the preceding Sample phase, because the responses were on opposite 
levers, reflects a "detachment" or dissociation of this aspect of ensemble activity from the "real-time" occurrence of the behavioral response. Moreover, Root 4 appears to have "preserved" an indication of the previous Sample phase response across the duration of the imposed delay intervals, providing a potential "bridge" for use in the Nonmatch decision process.

\section{Single-trial analyses of event-specific ensemble firing}

Root discriminant scores (Fig. $3 B$ ) for each of the behavioral events were analyzed on a trial-by-trial basis to determine which changes in ensemble firing extracted by the four significant roots occurred within DNMS trials. Root 1 (phase of task) and Root 3 (position of response), because they were directly related to the DNMS contingency, were the most directly involved in the "encoding" of DNMS task-relevant information. Figure 6 shows a two-dimensional (2-D) scatter plot of single-event discriminant scores for Roots 1 and 3, compiled across the seven different ensembles (animals). Each point in the scatter plot represents the intersection of the Roots 1 and 3 frequency histograms of discriminant scores (Fig. $3 B$ ) shown along their respective axes. Different trial types (i.e., L.Sample-R.Nonmatch), are represented by clusters of blue circles and squares, versus red symbols for the opposite trial type (R.Sample-L.Nonmatch). The clustering of the individual scores in each of the four quadrants of Figure 6 reflects the tendency for Roots 1 and 3 scores to discriminate complementary features of the same behavioral events on each DNMS trial. Both the compactness of the clusters and the extent of separation between them along both axes attest to the degree of discrimination by Roots 1 and 3 of these two task dimensions within a trial. The clustering of the two event scores from the same trial within diagonal quadrants reflects the consistent representation of events in register with the Nonmatch "decision rule" operating on each trial and confirms that the overall mean discriminant scores for each of these roots shown in Figure 5 accurately reflected differential ensemble activity associated with single-trial events.

To determine how the actual patterns of neural activity in the ensemble differed, root-adjusted spatiotemporal firing surfaces (Fig. 3C) of ensemble activity were constructed for each behavioral event; Figure 7 shows the adjusted firing surfaces for Roots 1 and 3 obtained from a different animal (than shown in later figures) to illustrate the difference in firing patterns between animals. Because Root 1 extracted variance associated exclusively with differences between the Sample and Nonmatch phases of the task, the adjusted firing surfaces (Fig. 7, left) were quite similar with respect to left and right lever presses in either phase of the task. In marked contrast, the adjusted firing surfaces for Root 3 in Figure 7 did not change appreciably as a function of Sample versus Nonmalch phase, but exhibited distinct differences with respect to the position of the lever response (Fig. 7, Root 3, L vs R). The upper and lower panels in Figure 7, therefore, show distinctions between adjusted firing surfaces with respect to Roots 1 and 3 in relation to phase and lever position. However, even though the spatiotemporal firing patterns for individual animals were not the same (see Fig. 8), the behavioral encoding processes in the ensembles were the same, and different patterns of activity within animals, therefore, were distinguished by the same canonical roots extracted for a given behavioral dimension. This indicated that it was necessary to consider the collective activity of the entire ensemble to identify all of the relevant encoded behavioral informa- tion and that such information could not be determined by separate examination of individual neuron activity.

Because each canonical root represented an independent source of variation in ensemble activity, the simultaneous influence of Root 3 (position) with Root 1 (phase) on ensemble firing in the same DNMS trial accounted for $66 \%$ of the overall variance on correct trials across all animals. The combined effects of Roots 1 and 3 , therefore, were considerable and were consistent with changes in ensemble activity within the DNMS trial in accordance with the "Nonmatch decision rule," indicated by the diagonal clusters of discriminant scores in Figure 6. The remaining two significant sources of variance extracted via the canonical discriminant analysis, Roots 2 and 4, likely were also involved in these same events on individual trials. However, Root 2 appeared to discriminate processes that were only active in the Nonmatch phase of error trials and will be discussed below in that context. Root 4, which extracted scores for Sample lever presses and did not change status during the Nonmatch phase, also showed individual trial variation in accordance with the position of the lever press. Next it was necessary to verify that the encoded patterns in Roots 1 and 3 were functionally relevant to performance of the DNMS task by assessment of these same patterns on error trials.

\section{Error-generating processes in spatial DNMS performance extracted from ensemble activity}

The canonical discriminant analysis provided the means to examine the lype of encoding or representation present during the Sample phase preceding the commitment of errors in the DNMS task. It was determined that two distinct types of encoding differences were associated with error (i.e., "match") responses in the Nonmatch phase of the task. These differences in root patterns were not present on correct trials and reflected aspects of ensemble activity that failed to distinguish correctly behavioral events encoded during the Sample phase. It was possible, therefore, to "classify" error trials independent of behavioral performance on the basis of "inappropriate" ensemble firing.

\section{Ensemble miscoding errors in the Sample phase}

Examination of differences in Root 3 on correct and error trials provided a key breakthrough in understanding the relationship between Sample phase ensemble activity and decision processes in the Nonmatch phase of the DNMS trials. Because there were no differences in Root 1 discriminant scores with respect to correct versus error trials, the only possible basis for generating errors was the Root 3 ensemble activity "encoded" in the Sample phase. Root 3 extracted features of ensemble activity related to position of the lever press; thus, comparison of adjusted firing surfaces provided a means of determining retrospectively the accuracy and strength of the encoding for this root in the Sample phase on error versus correct trials. Figure $8, A$ and $B$, shows that the adjusted Root 3 ensemble firing surfaces, for the animal whose data are shown in Figure 4, on error trials were similar to the surfaces generated on correct trials. Quite unexpectedly, however, it was determined that the Sample phase Root 3 firing surfaces on error trials did not correspond to the actual lever pressed when compared with surfaces generated on correct trials (Fig. 8A,B), but they were appropriate for the opposite nonpressed lever. Thus, in contrast to correct trials, on error trials the pattern in Root 3 revealed that the ensemble had actually "miscoded" the position of the Sample phase behavioral response (Fig. $8 B$ ). Consequently, in the Nonmatch phase of an error trial, the behavioral response was a "match" instead of a nonmatch of the position of the Sample 


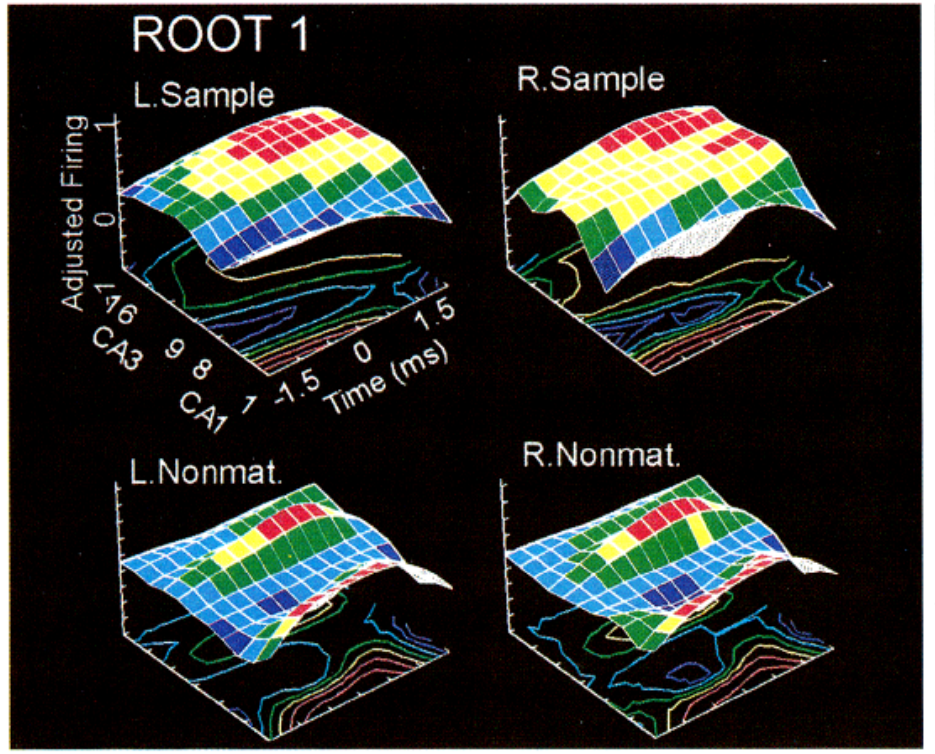

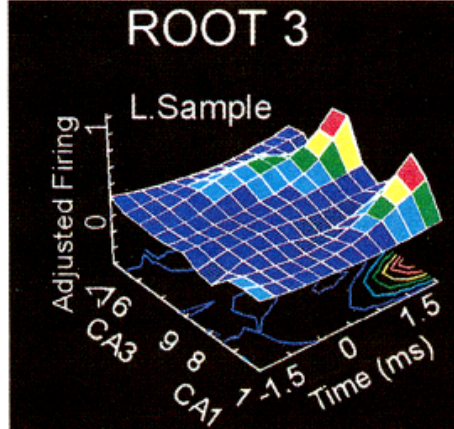

L. Nonmat.

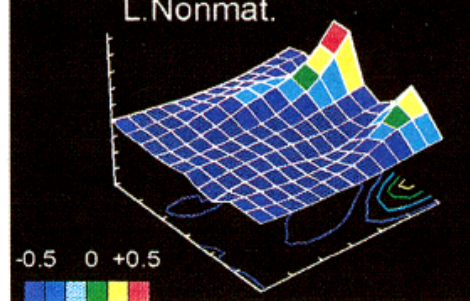

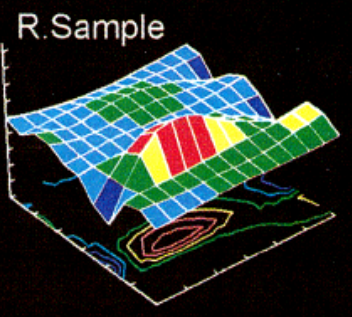

R.Nonmat.

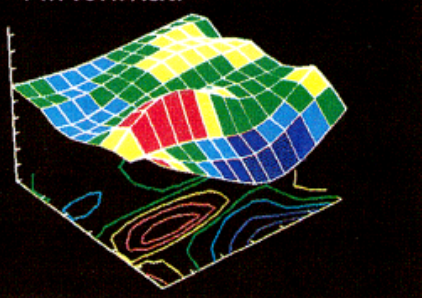

Figure 7. Top of page. Adjusted Root 1 and 3 firing surfaces. The adjusted 3-D firing "surfaces" were calculated as in Figure $3 C$ from mean firing rate for all correct trial events ( $\sim 100$ trials each) and were adjusted by either Root 1 or Root 3 coefficients. In both cases illustrated, surfaces that were diagonal to each other occurred on the same DNMS trial (i.e., Sample and Nonmatch). For a given behavioral event, the patterns for Roots 1 and 3 reflect simultaneous but independent firing tendencies adjusted by the respective roots, as evidenced by the different spatiotemporal regions involved in generating the surfaces. Color scale indicates adjusted firing rate from $<-0.5 \mathrm{~Hz}$ (dark blue) to $>+0.5 \mathrm{~Hz}$ (red) in the same increments as the black and white scale in Figure 3C. The gray shading seen on some surfaces (e.g., R.Sample, Root 1) represents the "underside" of the firing surface. Color contour lines are scaled the same as the surface colors and reflect changes in the surfaces above. Time is plotted relative to the lever press response (time $=0$ ): neurons are sorted according to position on the recording array (1-16). Labeling of scales and axes is the same as in Figure 3C.
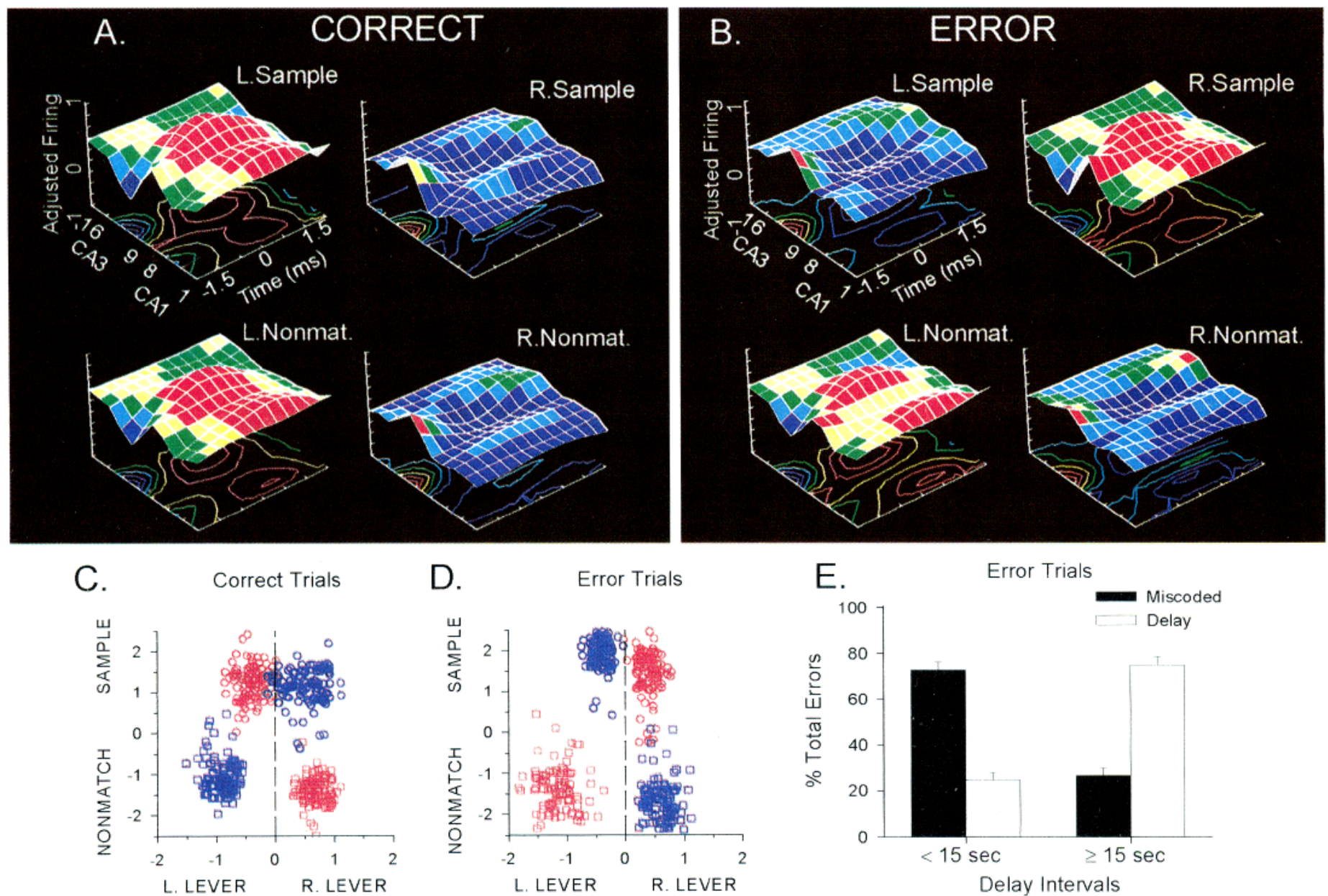

D. Error Trials

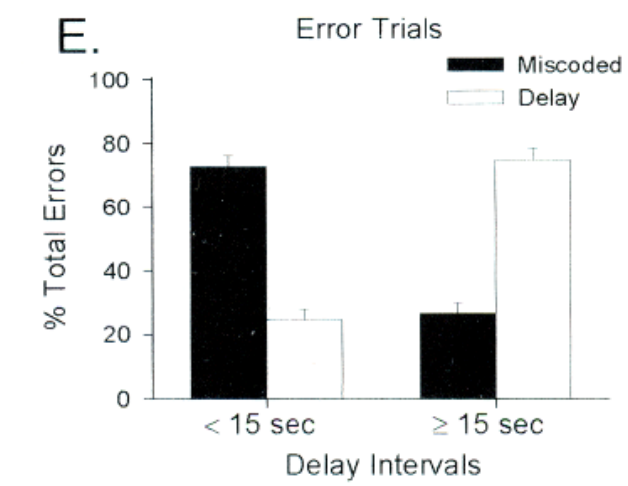

Error Trials

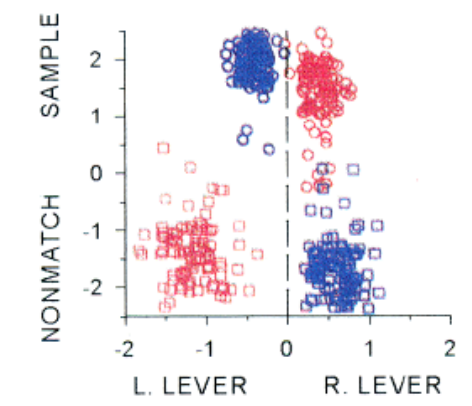


phase response. The presence of miscoding in Root 3 in the Sample phase suggested that it was the ensemble "representation" and not the behavioral response that served as the basis for the decision in the subsequent Nonmatch phase of the trials.

The DNMS performance curve (Fig. $1 B$ ) shows that there was an overall $25 \%$ drop from maximum ( $92 \%$ ) accuracy across delays of 1-30 sec. Because the presence of miscoded Sample information in the ensemble was never associated with a correct response on that trial, it is likely that the decision on error trials involved miscoded information to generate the response in the Nonmatch phase. However, it is important to note that application of the Nonmatch decision rule to miscoded Sample phase information would in fact generate a "match" or error response, irrespective of delay, in the Nonmatch phase. Comparing the number of trials across all animals in which miscoding in Root 3 was detected with the total number of error trials $(n=1795)$ revealed a large percentage $(40 \%)$ of errors due to miscoding $(n=671)$. Miscoding was present on $9.3 \pm 0.8 \%$ of total trials $(n=7162)$, and in no instance was miscoding detected on correct trials at any delay. However, on error trials with delays of $<15 \sec (n=463), 73 \%$ $(n=338)$ of all errors were associated with miscoding in Root 3 (Fig. $8 E$ ). On error trials with delays $\geq 15 \mathrm{sec}(n=1332)$, miscoding was present on only $25 \%(n=333)$ of error trials primarily because of the increase in frequency of other types of errors (described below). The scatter plots of single-trial discriminant scores in Figure 8, $C$ and $D$, show that error trials with delays $<15$ sec were nearly always associated with miscoding in Root 3 during the Sample phase, as indicated by the reversed positions of the colors (blue and red) relative to correct trials.

Miscoding errors should not be "delay-dependent" because the miscoded information is incorporated during the Sample phase before exposure to the delay interval. The presence of miscoding errors, therefore, suggests that events from the previous trial may "proaclively interfere" with the encoding of information in the Sample phase on the current trial. This possibility was supported by the determination of a significant $\left(F_{(1,239)}=9.24, p<0.001\right)$ difference in performance between similar (same) and dissimilar (different) preceding DNMS trial types (Fig. 9). When the preceding DNMS trial was the same as the current trial, performance accuracy was significantly improved compared with trials that were not the same. The mean percentage of trials in which such proactive interference was detected $(8.5 \%)$ was similar to the mean pcrcentage of trials $(9.3 \%)$ on which miscoding by Root 3 was ascertained across all animals.

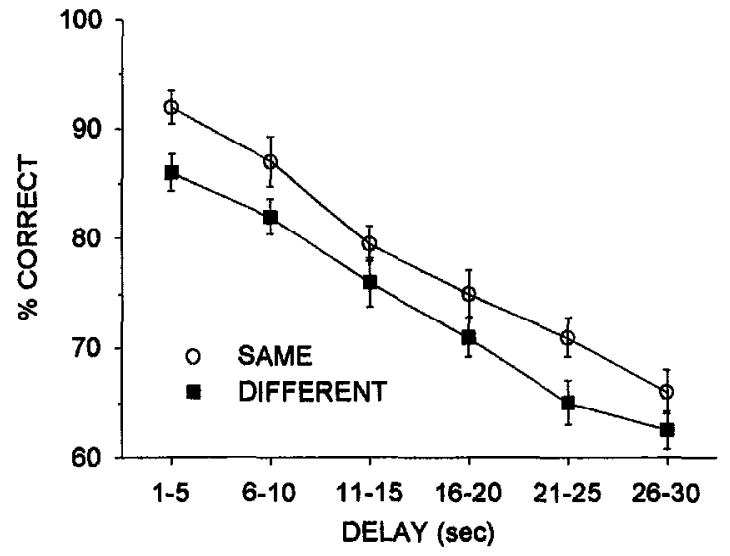

Figure 9. Effect of proactive interference from the preceding trial on DNMS performance. Mean $( \pm S E M)$ percent correct performance is plotted for all DNMS trials across all seven animals as in Figure $1 B$. Trials were sorted on the basis of whether the preceding trial was the same or different with respect to lever position in the Sample phase. Thus, a sequence of two L.Sample-R.Nonmatch trials was classified as $S A M E$, whereas a sequence of R.Sample-L.Nonmatch, L.Sample-R.Nonmatch trials was classed as DIFFERENT. The parallel nature of the two curves illustrates the lack of delay-dependent influence of proactive interference. Axes are the same as in Figure $1 B$.

\section{Delay-dependent errors in hippocampal ensemble codes}

Miscoded hippocampal ensemble activity in the Sample phase, therefore, could not be the basis for the delay-dependent deficit depicted in the DNMS performance curve (Fig. $1 B$ ), because miscoding would produce an equal proportion of errors at all delays (Fig. 9). Sixty-four percent of all errors were related to performance decrements at longer ( $\geq 15 \mathrm{sec}$ ) delay intervals. To determine the relationship to ensemble activity, discriminant scores and adjusted firing surfaces for Roots $\mathbf{1}$ and $\mathbf{3}$ on error trials with delays $\geq 15 \mathrm{sec}$ were compared with those from correct trials (Fig. 10A,B). On correct trials with delays $\geq 15 \mathrm{sec}$, the adjusted firing surfaces for Root 3 (Fig. 10A) were nearly identical to correct trials with delays $<15 \mathrm{sec}$ (see Fig. $8 A$ ). Scatter diagrams of discriminant scores for these correct trials (Fig. 10C) were clustered more distinctly than on trials with delays $<15 \mathrm{sec}$ (Fig. $8 C$ ). Alternatively, Figure $10, B$ and $D$, shows that the adjusted firing surfaces were more similar and scatter plots more overlapped for Root 3 on error trials with delays $\geq 15 \mathrm{sec}$, for both left

\section{$\leftarrow$}

Figure 8. Bottom of page. Adjusted firing surfaces from a single animal for Root 3 on correct versus error trials with delays $<15$ sec. $A$, Correct: Root 3-adjusted firing surfaces were distinct for left versus right Sample lever positions regardless of task phase (Sample vs Nonmatch) on correct trials. In each set of four surfaces, left lever responses are shown on the left, with right lever responses on the right; thus, the surface for the appropriate Nonmatch response is always represented as diagonal to the Sample response. On correct trials, the surface patterns changed between Sample and Nonmatch phase, indicating that the adjusted Root 3 firing pattern also changed to reflect the switch from left to right responses within each trial. Calculation of surfaces, axis scales, event labels, and color scale are the same as in Figure 7. B, Error: Root 3-adjusted firing surfaces in the Nonmatch phase on error trials were similar to those in the Nonmatch phase of correct trials $(A)$. The Sample phase surfaces were exactly the same as on correct trials, but reversed with respect to position of the response. Because the Nonmatch response was on the same lever as the Sample response (i.e., a "match" response), surfaces in the same column (not the diagonal) reflect within-trial events (i.e., left-left) on error trials. Note that even though the position of the response did not change, the adjusted firing surface did change between the Sample and Nonmatch phases. $C, 2$-D scatter diagram of Root 1 versus Root 3 exhibits four distinct clusters corresponding to behavioral event classifications for correct trials with delays $<15 \mathrm{sec}$. Events within the same type trial are shown in the same color (red, L.Sample-R.Nonmatch; blue, R.Sample-L.Nonmatch). Note that events within a trial are represented diagonally. Axis scales and labeling are the same as in Figure 6. $D$, Scatter diagram of Root 1 versus Root 3 discriminant scores for error trials with $<15$ sec delays. Individual scores and trials are color-coded as in $C$; however, note the reversal of color-coded clusters. Because the horizontal axis indicates the discriminant score values, scores from the same trials are still represented on the diagonal (i.e., miscoded) even though Sample and Nonmatch responses were on the same lever. E, Source of behavioral errors at different delays is shown as percentage of total errors. Root 3 discriminant scores were examined for miscoding during the Sample phase, to compare the percentage of miscoded trials to all trials with behavioral errors, and were sorted by length of delay ( $<15 \mathrm{vs} \geq 15 \mathrm{sec}$ ). Miscoding in the Sample phase was never associated with correct trials; thus, behavioral crrors werc sorted further according to whether miscoding occurred. Behavioral errors not associated with miscoding subsequently were termed "delay-dependent" (Delay) errors. 

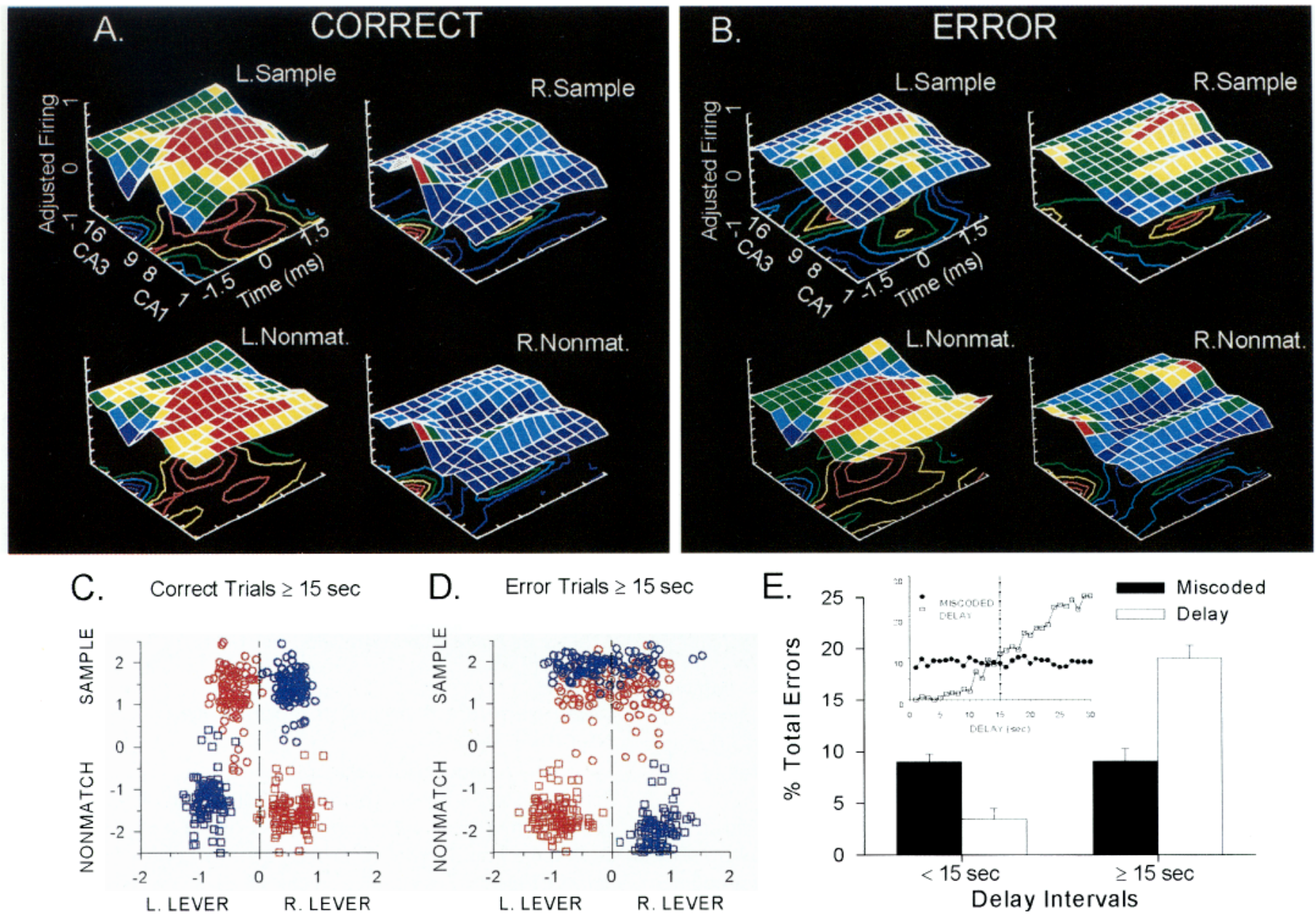

Figure 10. Adjusted firing surfaces for Root 3 on long delay ( $\geq 15 \mathrm{sec}$ ) correct and error trials. A, Correct: Root 3 adjusted firing surfaces (from the same animal as in Fig. 8) for correct trials at long delays ( $\geq 15 \mathrm{sec}$ ) were nearly identical to correct trials with delays $<15 \mathrm{sec}$. Axes, scales, and events are the same as in Figure 8A. B, Error: Root 3 surfaces in columns represent within-trial changes in firing related to error trials (match responses). Note the distinct encoding of Nonmatch lever position, although the surfaces for the left and right Sample responses (L.- and R.Sample) were considerably less distinct from each other. Axes, scales, and events are the same as in Figure $8 B$. C, Scatter diagram of Root 1 versus Root 3 single-trial discriminant scores for long ( $\geq 15 \mathrm{sec}$ ) delay correct trials. Discriminant scores were distributed into four distinct clusters similar to correct trials at short $(<15 \mathrm{sec})$ delays (Fig. 8C). D, Scatter diagram of Root 1 versus Root 3 single-trial discriminant scores for long ( $\geq 15$ sec) delay error trials. Root 3 discriminant scores were distinctly clustered in the Nonmatch, but not the Sample, phase. The mixed pattern of discriminant scores in the Sample phase would produce the similar appearing 3-D firing surfaces for both lever positions shown for a single animal in $B$. Axis scales and labels are the same as in Figure $8 D$. E, Percentage of total trials that were miscoded versus delay-dependent (Delay) errors was calculated for trials with $<15$ versus $\geq 15$ sec delays. Inset, Percentage of error trials across delays plotted in $1 \mathrm{sec}$ increments. Delay-dependent crrors ( $\square$ ) consistently increased as length of delay increased (from 10 to $30 \mathrm{sec}$ ). Percentage of miscoding errors $(\bullet)$ did not change across all delay intervals $(1-30 \mathrm{sec})$.

and right Sample lever presses. As with delays of $<15 \mathrm{sec}$, these same measures in the Nonmatch phase were nearly the same as on correct trials. Again, there were no differences in Root 1 discriminant scores or surfaces between error and correct trials with delays $\geq 15 \mathrm{sec}$. The lack of discrimination by Root 3 in the Sample phase on error trials with $\geq 15 \mathrm{sec}$ delays, therefore, indicates that lever position encoding was homogeneous or inconsistent.

Figure $10 E$ shows that the proportion of error trials that did not exhibit miscoding was quite low at short delays, yet increased linearly on trials with longer delays, suggesting that errors that were not the result of miscoding were "delay-dependent." This was confirmed by examination of the distribution of discriminant scores for Sample phase responses as a function of delay interval on correct versus error trials (mean \pm SEM scores) in Figure 11, $A$ and $B$. As the duration of the delay interval increased, the means for Root 3 discriminant scores remained significantly sep- arated on correct trials but "converged" toward values that indicated lack of discrimination (the midline, 0.0 ) on error trials. This reduction in Root 3 discriminant scores reflected "nondiscriminated" or "weakly discriminated" Sample phase response position information as a function of trials with increasing delay intervals. Figure $11 B$ shows that at delays of $\geq 20 \mathrm{sec}$ the means of the discriminant scores on error trials lic on or near the midline of the scatter plot and represent "nondistinct encoding" (i.e., lack of separation) of the position of the Sample response. This convergence of discriminant scores on long delay error trials, therefore, was indicative of three types of non-optimal encoding in Root 3: (1) miscoding, (2) nondiscriminated position (scores that were on the midline), and (3) correct but "weakly" (nonsignificantly) discriminated response position. Because the latter two types of encoding were not associated with errors on short delay error trials (Fig. 11B), they must have been associated with correct trials at short delays, but subsequently became "at risk" as the length of 


\section{A. Correct Trials}

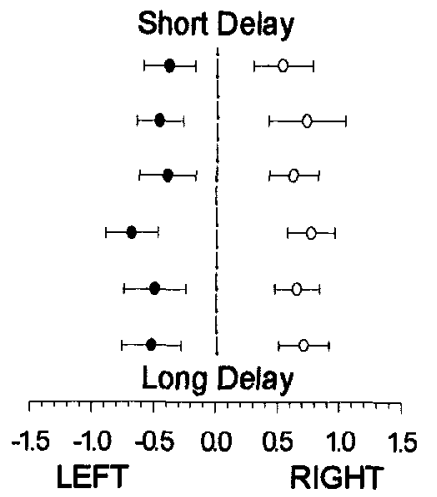

B. Error Trials

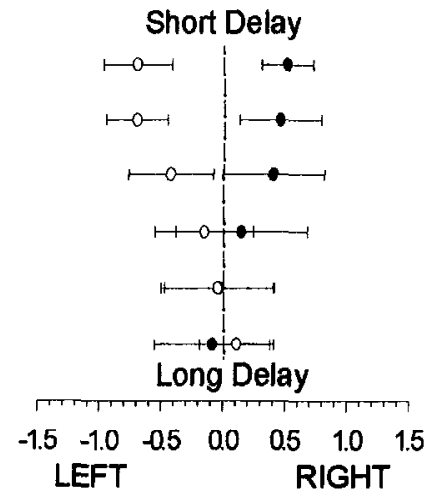

Figure 11. Distribution of left and right Root 3 Sample scores across different delay intervals for correct and error trials, $A$, Mean ( \pm SEM) Root 3 discriminant scores for all correct Sample phase responses (across animals) are plotted according to lever position and length of delay. Scores were sorted by delay in $5 \mathrm{sec}$ increments (see $x$-axis of Fig. $1 B$ ) from 1-5 sec (short delay) to 26-30 sec (long delay). Rool 3 scores were significantly discriminated (separated) with respect to left (negative scores) and right (positive scores) lever position on correct trials at all delays. (O), Left lever responses; $(O)$, right lever responses. $B$, Mean ( \pm SEM) Root 3 Sample phase discriminant scores across delays for error trials. Scores converged toward midline and completely overlapped (i.e., not significantly discriminated) at longer delays. Lever position miscoding on trials with delays $<20$ sec is shown by the reversal of solid (left) and open (right) symbols. C, Frequency distribution of Root 3 Sample phase discriminant scores on short delay trials. Distinct positive and negative "peaks" (white bars) corresponded to right and left lever position, respectively. The large central peak (black bars) corresponded to discriminant scores that were not significantly different (left or right; $p>0.10$ ). Horizontal scale, Root 3 discriminant score, see $x$-axis in Figure $10 C, D$; vertical scale, frequency of occurrence of discriminant score value across all trials and animals. $D$, Discriminant scores for long delay correct trials show distinct positive and negative peaks (white bars), but marked reduction in nonsignificantly discriminant scores (black bars). $E$, Frequency distribution of Root 3 scores on short delay error trials show reduced miscoded (diagonal pattem) positive and negative peaks, but only a few scores were not significant (black bars). Decreased overall magnitude results from lower percentagc of total trials. $F$, Root 3 Samplc phase scorcs for long delay ( $\geq 15 \mathrm{sec}$ ) error trials show marked increase in nonsignificant scores (black bars) on long delay error trials. Positive and negative peaks are due to the presence of both miscoded trials (cross-hatched) and correctly encoded trials (diagonal stripes). Note that the number of miscoded trials is similar on error trials at short and long delays but correctly encoded trials appear only on errors with long delays ( $E$ vs $F$ ). the delay interval increased. Hence, less than optimal Sample phase encoding by Root 3 was a potential factor in determining the delay-dependent decrement in accuracy reflected in the DNMS performance curve (Fig. $1 B$ ).

The lack of clustering and high degree of overlap in the discriminant scores for Root 3 in Figure $10 D$ reflect the above three types of circumstances acting on long delay error trials. Because length of delay interval was not a factor at the time of the Sample response, the same type of nondiscriminated and weakly discriminated Sample phase responses should be present on nonerror trials; otherwise a "unique" type of encoding would have to have occurred "exclusively" on error trials at long delays (when that knowledge was not available to the animal). Figure 11, $C$ and $D$, shows frequency histograms of the distribution of Root 3 discriminant scores for correct and error trials at long and short delays. The white bars represent the frequency of scores that met the criterion for significant discrimination by Root 3 . These are identified as separate "peaks" for the left and right Sample phase responses. Black bars reflect Root 3 scores that either were not discriminated or were weakly discriminated during the Sample phase of the task on short delay correct trials. Examination of correct trials at delays $\geq 15 \mathrm{sec}$ in Figure $11 \mathrm{D}$ reveals a distinct absence of the latter types of scores and an enhanced separation (i.e., encoding) of the "peaks" for left and right lever discriminant scores. In Figure 11, $E$ and $F$, the frequency histograms of Root 3 discriminant scores on error trials with delays $<15$ or $\geq 15 \mathrm{sec}$ are also shown. Clearly, for delays $<15 \mathrm{sec}$, miscoding errors predominated (diagonally striped bars). However, on error trials with delays $\geq 15 \mathrm{sec}$, the frequency of nondiscriminated or weakly discriminated scores increased markedly as shown by the black bars in Figure $11 F$. The cross-hatched area reflects the combined presence of miscoded and correctly encoded trials at long delay intervals.

Thus, the same nondiscriminated or weakly discriminated Root 3 scores that were associated with correct trials at short delays (Fig. 11C) were transformed to error trials at long dclays (Fig. $11 F$ ). The Sample phase events not significantly encoded by the ensemble, therefore, were identified retrospectively as being at risk for producing errors on long, but not on short, delay trials. Such a transition would account for the lack of errors other than miscoding at short delays, as well as the "delay-dependent" nature of the other class of behavioral errors reflected in the DNMS performance curve (Fig. 1B). Whether nondiscriminated or weakly discriminated Sample phase responses in Root 3 accounted exclusively for the disproportionate increase in errors at long delays was addressed by comparing the proportion of these 


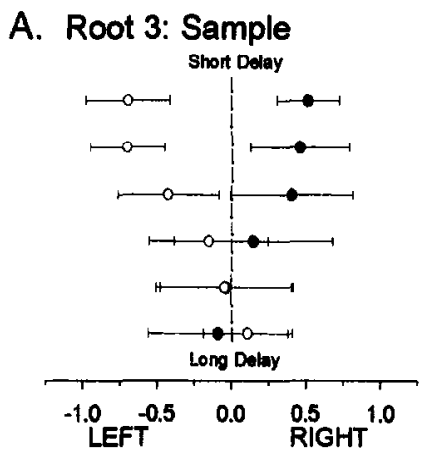

B. Root 3: Nonmatch

Figure 12. Within-trial differences in distribution of mean $( \pm$ SEM) Root 3 and Root 4 discriminant scores across delays in Sample versus Nonmatch phases of DNMS task on error trials. $A$, Root 3 Sample phase mean discriminant scores show miscoding at short delays but converged to the midline at longer delays (scores are plotted as in Fig. 11B). (O), Left lever responses; (O), Right lever responses. $B$, Root 3 mean $( \pm \mathrm{SEM})$ discriminant scores in the Nonmatch phase appropriately encoded lever position at all delays. $C$, Root 4 mean discriminant scores are significantly discriminated (separated) during the Sample phase on error trials at all delay intervals $(1-30 \mathrm{sec})$. $D$, Root 4 mean scores converge during the Nonmatch phase and were nonsignificant with respect to lever position in the Nonmatch phase at delays $\geq 15 \mathrm{sec}$.

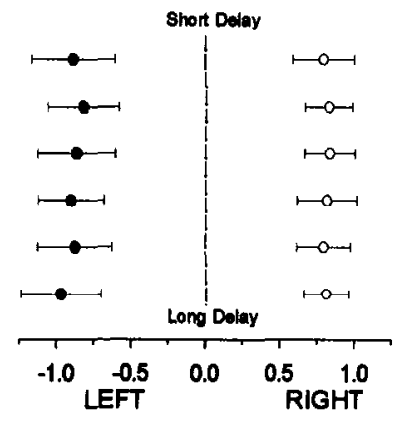

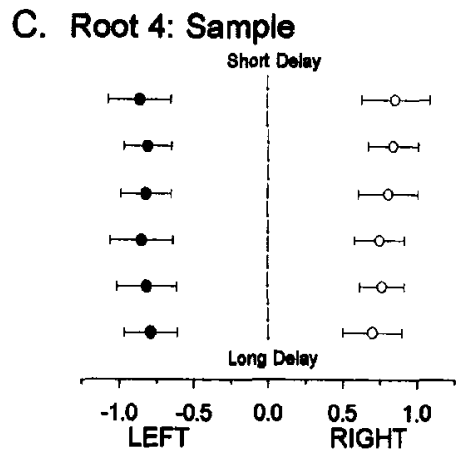

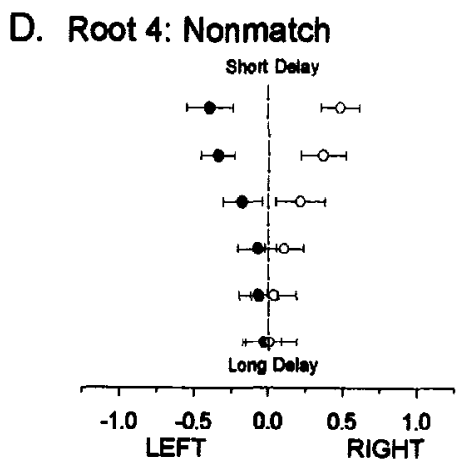

two occurrences on short versus long delays. The proportion of such weakly or nonencoded trials (Fig. 11B) on short delay correct trials versus long delay error trials was 12 vs $14 \%$ of total trials, respectively. In addition, the percentage of total trials attributable exclusively to errors other than miscoding (Fig. 11E,F) and the percentage of correct trials that were nondiscriminated or weakly discriminated in the Sample phase (Fig. $11 C, D$ ) were the same. Thus, the population of nondiscriminated or weakly discriminated Sample phase responses shifted from being associated with correct trials at short-delays to being associated with error trials at long delay intervals $\left(F_{(2,239)}=3.91, p<0.01\right)$, providing the basis for the delay-dependent nature of DNMS performance accuracy.

\section{Roots 3 and 4 reflect a common decision process}

As stated earlier, Root 4 discriminant scores on all correct trials were specific to the position of the Sample phase lever press in both the Sample and Nonmatch phases of the task (Fig. 5). The representational significance of the scores in Root 4 are not completely understood because of two observations: (1) Root 4 appeared to encode the Sample lever press accurately on short delay error trials (i.e., miscoded trials), which obviously did not lead to correct responding; and (2) the reappearance of the Root 4 "pattern" in the Nonmatch phase was completely dissociated from the physical position of the response. Because Root 4 accurately "reproduced" the (opposite) response position information from the previous Sample phase of the same trial (Fig. 5), appropriate Sample phase information in Root 4 was present in the overall ensemble activity in the Nonmatch phase on all correct trials. Thus, at the time of the Nonmatch lever press on correct trials, information extracted in Roots 3 and 4 together represented both elements of the appropriate Sample and Nonmatch "firing patterns" coincident with the time $( \pm 1.5 \mathrm{sec})$ of the decision to make the response in the Nonmatch phase.

The question remains as to how information in Root 4 was related to Root 3, if at all. A partial answer was obtained by examining mean discriminant scores for Roots 3 and 4 on error trials (Fig. 12). Figure 12, $A$ and $C$, shows that with delays of $\geq 15$ sec Root 4 patterns were appropriately segregated according to the position of the Sample phase response (similar to correct trials, Fig. $12 C$ ), whereas Root 3 scores converged toward the midline (lack of discrimination, Fig. 124). In the Nonmatch phase of the same error trials, however, Root 4 scores converged toward the midline in exactly the same manner as Root 3 Sample scores (Fig. 12D). As in other error trials, Root 3 scores in the Nonmatch phase accurately represented the position of the response even though the response was not appropriate (Fig. 12B). Therefore, across the delay interval there was a transition in Root 4 scores such that at the time of the Nonmatch response the "retained" Sample phase information was no longer distinct as on correct trials at the same delays (Fig. 12D). The degree to which this convergence in Roots 3 and 4 occurred was dependent on the length of delay and seemed to affect both roots in the same manner. This covariation with the status of information encoded in Root 3 suggested that Root 4 interacts at the level of a common process with Root 3 to alter activity in the ensemble during the delay, such that some fragment of Sample phase information is available at the time of implementation of the Nonmatch decision rule (in the Nonmatch phase).

\section{Root 2 Is sensitive to the Increased error on long delay trials}

The grcatly increascd ambiguity on long delay error trials in the distribution of discriminant scores in Roots 3 and 4 (Figs. 8, 10-12) during the Sample and Nonmatch phases may have reflected the lack of a functionally significant change in ensemble firing at the time of the response in the Nonmatch phase. The lack of distinct representation (separated clusters) of lever position information by Roots 3 and 4 may have led to responding on a chance basis (i.e., "guessing") on long delay error trials. If random responding were more prominent on long delay error trials, Root 2 , which was the only root that represented variance extracted exclusively on error trials (Fig. 5), possibly would be sensitive to 


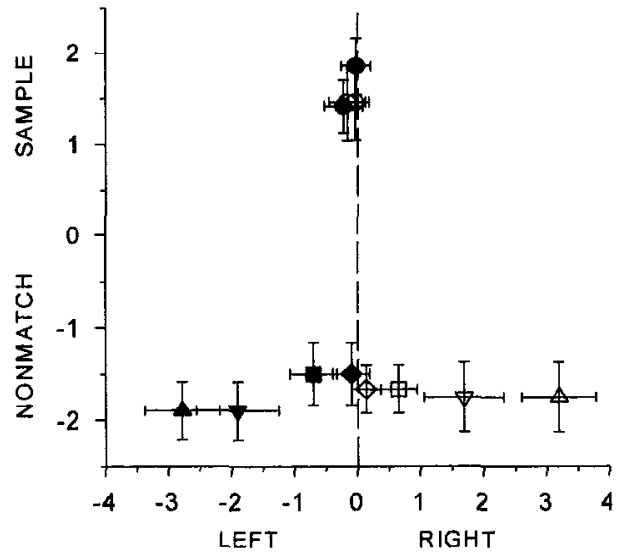

Figure 13. Root 1 versus Root 2 mean discriminant scores for correct and error trials, plotted as 2-D "centroids" (i.e., mean \pm SEM with respect to both Root 1 and Root 2). Sample phase discriminant scores were not significantly discriminated (as shown by all symbols on the midline). There were also no significant differences with respect to short $(<15 \mathrm{sec})$ or long delay ( $\geqq 15 \mathrm{sec}$ ) for Sample phase scores $(O)$. Error responses in the Nonmatch phase on trials with $\geq 15$ sec delays $(\triangle)$ were discriminated more significantly by Root 2 than at delays of $<15 \sec (\nabla)$. In addition, left and right centroids for correct Nonmatch responses at long delays ( $\square$ ) were also significantly $(p<0.05)$ different (separated) from each other. Scores for correct Nonmatch responses on short delay trials $(\diamond)$ were not significantly discriminated by Root 2 . Solid symbols, Left lever responses; open symbols, right lever responses.

this shift in strategy. Figure 13 shows that on error trials with $\geq 15$ sec delays, there was a significant increase $\left(F_{(1,1081)}=23.9, p<\right.$ 0.001 ) in separation of Root 2 mean discriminant scores compared with error trials at $<15 \mathrm{sec}$ delays. As shown earlier (Fig. 5), Root 2 scores did not differ significantly from zero for Sample responses (at short or long delays) or for correct responses in the Nonmatch phase at short delays (Fig. 13). The source of variance extracted by Root 2, therefore, was not present during events in which random responding was not likely (miscodes), but increased on long delay errors when accurate encoding of Sample information and its successful retrieval were poor. Surprisingly, additional support for this assumption was provided by the fact that Root 2 Nonmatch discriminant scores on long delay correct trials were also significantly $\left(F_{(1,1081)}=6.65, p<0.05\right)$ increased with respect to short delay correct trials (Fig. 13). If Root 2 reflected a process that accounted for errors of any type, including random responding, that process would also be expected on a small number of trials that are "guessed" correctly, as well as in error trials.

\section{Relationship of spatiotemporal firing to canonical roots}

The spatiotemporal contributions to Roots 1-4 were analyzed separately to determine whether the four identified sources of variance were distributed differentially with respect to particular anatomic or temporal domains of the recording array. The contribution of spatial and temporal factors was calculated using the coefficients of the linear equations ( $W_{n, t}$ in Fig. $3 A$ ), normalized as the sum of the absolute value of the individual coefficients divided by the sum of the absolute value of all coefficients for each ensemble. For temporal assessments, the $\pm 1.5 \mathrm{sec}$ time epoch around each behavioral event was subdivided into three $1 \mathrm{sec}$ periods, commencing $1 \mathrm{sec}$ before the lever response $(-1.5$ to $-0.5 \mathrm{sec}), \pm 0.5 \mathrm{sec}$ before and after the response, and $1 \mathrm{sec}$ after the response $(0.5-1.5 \mathrm{sec})$. There were slight differences $\left(F_{(2,18)}>\right.$ $5.82, p<0.05$ ) in percent contribution (Table 1, asterisks) across
Table 1. Percentage of canonical root accounted for by temporal segments of analysis period

\begin{tabular}{llll} 
& $\begin{array}{l}\text { Preresponse } \\
-1.5 \text { to }-0.5 \mathrm{sec} \\
(\%)\end{array}$ & $\begin{array}{l}\text { Response } \\
-0.5 \text { to }+0.5 \mathrm{sec} \\
(\%)\end{array}$ & $\begin{array}{l}\text { Postresponse } \\
+0.5 \text { to }+1.5 \mathrm{sec} \\
(\%)\end{array}$ \\
\hline Root 1 & $39.8 \pm 5.1$ & $28.9 \pm 3.4$ & $33.8 \pm 2.3$ \\
Root 2 & $19.3 \pm 3.2^{*}$ & $31.2 \pm 2.5$ & $47.0 \pm 2.4^{*}$ \\
Root 3 & $29.9 \pm 4.9$ & $21.2 \pm 4.1^{*}$ & $49.0 \pm 4.5^{*}$ \\
Root 4 & $33.6 \pm 4.7$ & $33.4 \pm 4.7$ & $33.2 \pm 2.7$
\end{tabular}

${ }^{*}$ Contribution significantly increased or decreased from equal distribution $(33.3 \%)$; $F_{(2,18)}>5.82, p<0.05$.

the three time domains, but none contributed exclusively to all of the variance extracted by any of the four Roots.

The possibility of differential contributions of specific neurons located in the anterior versus posterior (septo-temporal) CAl and CA3 hippocampal regions was also analyzed in a similar manner. Table 2 shows that the overall contribution to Roots 1-4 was slightly greater for CA3- vs CA1-located neurons within the ensemble. Changes in $\mathrm{CA} 3$ neuronal activity were most significant for Root $3\left(F_{(3,24)}=11.2, p<0.001\right)$ with moderate changes in CA1 neurons $\left(F_{(3,24)}>4.55, p<0.05\right)$ associated with Roots 1 and 4 (Table 2). Contributions to the overall variance in ensemble firing ranged from 42.9 to $57.1 \%$ as a function of position on the electrode array (anterior or posterior; Table 2). There was a slight trend toward increased contribution from the more septal hippocampal regions, but this was significant only for Root $1\left(F_{(3,24)}\right.$ $=6.1, p<0.01)$ and $\operatorname{Root} 3\left(F_{(3,24)}=5.6, p<0.01\right)$. When variance associated with anatomic location was analyzed according to both the CA1/CA3 fields and their anterior-posterior location, proportions ranged from 9.3 to $35.4 \%$ (Table 2) but were distributed fairly evenly across all regions with no distinct gradient. Thus, firing associated with each behavioral event underlying the extracted root variances did not derive solcly from a singlc anatomic region or temporal domain, but was distributed across all neurons within the ensemble. However, more fine-grained analyses might reveal subtle spatiotemporal factors organized along more subtle anatomic features of hippocampal architecture (Amaral and Witter, 1989; Witter et al., 1989; Amaral et al., 1991).

\section{DISCUSSION}

\section{Similarities across ensembles recorded in different animals}

The canonical discriminant analysis derived similar patterns of activity from ensembles of hippocampal neurons recorded in seven different animals. The sources of variance extracted and the calculated changes in ensemble activity obtained within different behavioral contexts were remarkably similar (Fig. 5). Two possible reasons for this are (1) similarity across animals in behavioral performance of the task (Fig. 1B) or (2) the electrode array placements sampled the same population of hippocampal neurons in each animal. The difference in adjusted patterns of neuronal firing within ensembles obtained across animals (Fig. 7) argues against the latter possibility. The similarity in DNMS performance across different animals is a more likely basis for this similarity, because all seven ensembles exhibited similar rank ordering of the four significant sources of variance (roots) associated with the behavioral event classification scheme. 
Table 2. Percentage of canonical root accounted for by different spatial locations within the hippocampus

\begin{tabular}{|c|c|c|c|c|c|c|c|c|}
\hline & \multicolumn{2}{|l|}{$\mathrm{CA} 1(\%)$} & \multicolumn{2}{|l|}{$\mathrm{CA} 3(\%)$} & \multicolumn{2}{|c|}{ Combined $(\%)$} & \multicolumn{2}{|c|}{ Combined $(\%)$} \\
\hline & Anterior & Posterior & Anterior & Posterior & CAl & $\mathrm{CA3}$ & Anterior & Posterior \\
\hline Root 1 & $23.1 \pm 4.7$ & $19.0 \pm 4.9$ & $33.1 \pm 5.4$ & $24.8 \pm 3.7$ & $42.1 \pm 5.5$ & $57.9 \pm 3.9$ & $56.2 \pm 5.8$ & $43.8 \pm 5.6$ \\
\hline Root 2 & $20.1 \pm 6.1$ & $26.6 \pm 5.1$ & $25.0 \pm 4.2$ & $28.3 \pm 5.1$ & $46.7 \pm 5.2$ & $53.3 \pm 4.4$ & $45.1 \pm 6.2$ & $54.9 \pm 5.1$ \\
\hline Root 3 & $20.7 \pm 5.5$ & $9.3 \pm 3.7$ & $35.4 \pm 5.0$ & $34.6 \pm 6.0$ & $30.0 \pm 5.7$ & $70.0 \pm 6.1$ & $56.1 \pm 8.7$ & $43.9 \pm 5.7$ \\
\hline Root 4 & $21.4 \perp 5.4$ & $23.0 \pm 3.3$ & 27.2 上 3.9 & $28.4 \pm 4.6$ & $44.4 \pm 5.3$ & $55.6 \pm 4.4$ & $48.6 \pm 5.2$ & $51.4 \pm 6.0$ \\
\hline
\end{tabular}

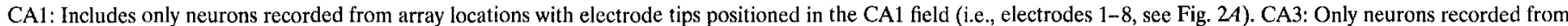

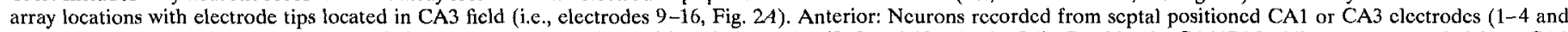

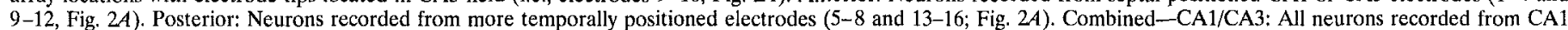

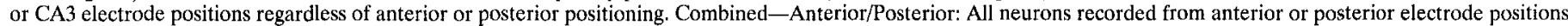
regardless of $\mathrm{CA} 1$ or $\mathrm{CA} 3$ positioning.

\section{Relevance of hippocampal ensemble activity to DNMS performance}

The findings presented here provide new insight into information representation and processing within the hippocampus during performance of delayed recognition memory tasks. DNMS and DMS tasks exploit these processes by interpolating delay intervals between the encoding and decision phases of the task, requiring retrieval of item-specific information to perform correctly. The delay-dependent nature of performance in the DNMS task, therefore, reflects the susceptibility of the above processes to perturbations in the Sample phase via defective encoding, and in the Nonmatch phase due to interference, decay, or corruption of information during the delay. However, no matter what relationship is demonstrated between ensemble activity and behavioral performance, an important and crucial condition is the necessity of the hippocampus for optimal performance of the DNMS task (Zola-Morgan et al., 1989a,b; Gaffan and Murray, 1992; Squire, 1992; Angeli et al., 1993; Cohen and Eichenbaum, 1993; Rawlins et al., 1993; Eichenbaum et al., 1994). We have recently completed a set of studies that show that the DNMS task used here is severely disrupted by ibotenate lesions in which complete removal of only hippocampus, and not other retrohippocampal areas, produced a delay-dependent deficit in lesioned animals over their prelesion performance (Hampson et al., 1995). At delay intervals of 1-3 sec, however, there was no significant difference between pre- and postlesion performance. The task, therefore, has been confirmed to be dependent on the integrity of hippocampal tissue using methods of hippocampal removal that spare fibers of passage (Jarrard and Davidson, 1994).

A second test of the relevance of the ensemble encoding demonstrated here is the degree to which it accounts for and is consistent with performance on individual trials. The use of canonical discriminant analyses provided a key insight into how information critical to task performance was represented in the hippocampal ensembles (cf. Shannon, 1948; Hamming, 1986). As shown in Figures 8, 10, and 11, the relationship between rootadjusted ensemble firing surfaces, discriminant scores, and behavioral performance was highly consistent on individual correct trials. This proved to be so reliable that it was possible to analyze retrospectively the encoded patterns on any trial and essentially "predict" $75 \%$ of the behavioral errors, leaving only $9 \%$ of performance on total trials that could not be predicted from the ensemble analysis. That such "predictions" could be made solely on the basis of the patterns of activity extracted by the various roots in the Sample phase validates the relevance of the sources of variance identified by each of the canonical roots.

A third important qualification that must be met for the rela- tionship between ensemble activity and DNMS performance to be accepted as "functionally significant" is the necessity to rule out firing correlates of nonspecific behavioral factors that inadvertently might be construed as representative of DNMS-related processes. In the present studies, this was controlled for in a number of different ways. First, hippocampal ensemble activity was shown to be phasic and maximally differentiated (increased or decreased) during the Sample and Nonmatch phases of the task (Fig. 4), presumably the times during which relevant ensemble activity would be critical (Hampson et al., 1993b; Heyser et al., 1993). Second, the ensemble firing patterns generated were specific for particular behavioral events in terms of their "task relevance" and independent of direct motor or sensory components of the executed responses, factors that, if extracted, would have been at variance with the DNMS dimensions stipulated in the analysis. Third, there were no major differences in the profile of extracted root contributions during three different subperiods of the \pm 1.5 sec analysis interval bracketing each behavioral event, as would be expected if firing were related to movement per se. Fourth, patterns of ensemble activity differed significantly when exactly the same behavioral responses were executed. The best example of this was "miscoding" by the ensemble of position information in the Sample phase (Fig. 8). There were no detectable differences in Sample phase response characteristics in trials that were miscoded versus those in which the same lever press was encoded correctly. In addition, the demonstration that the miscoded information was always associated with erroneous responses in the Nonmatch phase indicates that it was the pattern of ensemble activity, not the behavioral response, that was functionally relevant. Finally, because the animal had no way of detecting whether a behavioral response in the Sample phase was to be associated with an error or correct trial, there was no way in which differential motor movements, etc. could be generated to account for miscoding. The above factors, together with several others, make interpretations of the results based on differences in motor acts, sensory events, or other factors, less accountable for the complexity in the data.

\section{Error patterns in hippocampal ensembles reflect different types of disruption in the encoding process}

The above analysis of ensemble recording techniques revealed the presence of two types of error processes, each differentially responsible for decreased accuracy in performance of the DNMS task. Both processes were identified retrospectively from analysis and identification of encoded patterns in the Sample phasc on error trials. Miscoding of response position information in the Sample phase produced approximately the same degree of performance reduction at all delay intervals (Fig. 9). In these in- 
stances, hippocampal neural activity in the Sample phase was not only dissociated from behavioral responding (i.e., left vs right lever presses), but consistently "registered" a behaviorally inappropriate Root 3 ensemble firing pattern (Fig. $8 B$ ). Although from an intuitive perspective this finding is reasonable, the direct demonstration that a specifically identified brain process was consistently associated with the "wrong" behavioral response, thereby causing an erroneous response in another phase of the task, has very little precedent in previous neurobehavioral studies (cf. Wilson et al., 1990; Rothblat and Kromer, 1991).

Further analyses revealed that the miscoding was possibly due to interference from previous trials acting in a proactive manner on ensemble encoding in the Sample phase of the task (Fig. 9). Proactive interference was demonstrated by Dunnett (1989) using the original version of this task. In our findings, miscoding errors predominated at short delays $(<15 \mathrm{sec})$ simply because delaydependent errors did not occur on those trials (Figs. 8E, 10E). Thus, at delays $<15 \mathrm{sec}$, DNMS performance reduction was primarily the result of miscoding. Normally, of course, this type of error cannot be accounted for in delay-type recognition tasks without concomitant multineuron recording. Because of its differential predominance at short delays, it can appear to be part of the delay-dependent error-generating process, contributing to deficits at short delays (Fig. 10E). The presence of miscoding as a factor in DNMS task performance has not been evaluated in detail in either the primate or the rat DNMS literature; however, the shapes of many published DNMS delay curves indicate that performance is rarely at $100 \%$ even at very short delay intervals (cf. Zola-Morgan et al., 1989a,b, 1992; Gaffan and Murray, 1992; Rawlins et al., 1993; Eichenbaum et al., 1994). It is likely, therefore, that the same relationship between miscoding and delaydependent errors demonstrated here operates to some extent in all delay-dependent tests of memory.

Errors on all other trials not classified as miscoded were demonstrated to be delay-dependent (Figs. 11, 12). Lack of distinct (significant) encoding of the response in Root 3 in the Sample phase, and the corresponding loss of specificity of the pattern in Root 4 in the Nonmatch (decision) phase on long delay trials, were the major hippocampal ensemble correlates of delay-dependent errors (Figs. 10, 12). That such nondistinct encoding in the Sample phase (Root 3) was at risk at longer delays was demonstrated by the fact that short delay trials with similar encoding deficiencies were associated with correct performance (Fig. 11C). Consequently, the only factor that could have acted to enhance the probability that these encoding deficiencies would be associated with an error versus correct trial was duration of the intervening delay interval (Figs. 10-12). Figure $10 E$ bears this out by showing that the frequency of error trials that were not the result of miscoding in the ensemble, increased as a direct function of length of interposed delay interval after 7-10 sec. This demonstration satisfies the condition for delay-dependent behavioral errors in the DNMS performance curve and is consistent with the notion of differential decay, corruption, or disappearance of taskrelevant information within the ensemble over the delay interval (Figs. 10-12).

The correspondence between nondistinct encoding of information in the Sample phase and the susceptibility to delay-dependent impairment in DNMS performance accuracy is compatible with several recent theories and observations regarding the role of the hippocampus in recognition memory. Such theories actually stipulate that the information decay process in hippocampus requires repetition for more permanent representations to be established
(McNaughton and Morris, 1987; Rolls, 1991a; Miyashita et al., 1992; Eichenbaum et al., 1994; Gluck and Myers, 1995). Other reports of neuron data collected in delay-type tasks are relevant to this suggestion (Fuster and Jervey, 1982; Watanabe and Niki, 1985; Hampson and Deadwyler, 1994). However, few of the above formulations of hippocampal memory processes place the degree of emphasis on encoding as warranted by the findings presented here, which show that the distinction with which information is encoded by ensembles of hippocampal neurons is a primary factor responsible for successful retention across longer delay intervals (see Squire, 1992).

It should be noted that the process of information retrieval (i.e., recurrence of the same cnscmble pattern) was not the only process in the Nonmatch phase potentially affected by the lack of distinct encoding in the Sample phase of the task. Successful DNMS performance also required accurate implementation of that information to make the opposite response and satisfy the Nonmatch decision rule. Utilization, and not necessarily retrieval, of Sample phase information, therefore, was also susceptible to disruption by the duration of interposed delay. However, performance on miscoded trials argues against the latter as a basis for delay-dependent crrors, bccause of Sample phase information that was inaccurate, i.e., miscoded, was not discarded or "corrected" before execution of the inappropriate response in the Nonmatch phase on error trials. The fact that miscoded Sample responses were never associated with correct trials, therefore, is a strong indication that once encoded, Sample phase information was "used" in the same manner on error as on correct trials.

Because of the Nonmatch decision rule in the DNMS contingency, an optimal circumstance would be to have the encoded Sample phase pattern "available" at the time of the decision in the Nonmatch phase of the task. Curiously, the canonical discriminant analysis of ensemble activity revealed that Sample phase information was available, during the Nonmatch phase, in Root 4. Root 3 information in the Nonmatch phase could not perform this function because it merely "tracked" the location of the Nonmatch response whether correct or incorrect. However, as demonstrated in Figures 11 and 12, complementary variations in Roots 3 and 4 between the Sample and Nonmatch phases suggest that Sample information in Root 3 could have been transferred to Root 4 during the delay and could have been available in the Nonmatch phase of the task to provide a "real-time" representation for application of the Nonmatch decision rule. Support for this assumption was provided by the lack of distinct Sample phase encoding in Root 3 and corresponding loss in Root 4 representation in the Nonmatch phase on error trials at long delays (Fig. 12). 'l'hus, Root 4 information on error trials at long delays may have been corrupted by the disruption of the process responsible for transferring information from Root 3 during the delay (Fig. 12).

Ensemble discriminant scores for Root 2 (Nonmatch phase errors) were increased on long versus short delay error trials (Fig. 13). The presence of a significant factor within the ensemble firing patterns restricted to the Nonmatch response on error trials may have reflected the different sensory conditions present on error (lights out) versus correct (water delivery) trials. However, this explanation does not account for the "increased discrimination" by the Root 2 variance source in the Nonmatch phase on long versus short delay trials for both correct and error responses (Fig. 13). Implementation of a random responding or "guessing" strategy in the Nonmatch phase as a result of ambiguous (nondistinct) representations in Roots 3 and 4 on long delay trials is a possible 
alternative explanation that is consistent with the change in both correct and error trial scores for Root 2.

A small but important percentage (25\%) of long delay error trials was associated with distinct and appropriate encoding of the Sample response in Root 3 (Fig. $11 F$ ). However, this percentage of error trials represents $<10 \%$ of total trials and is well below expected chance levels $(50 \%)$ of responding in this task. Because the same trials that were associated with correct performance at short delays were shown to be at risk when longer delays were imposed (Fig. 11), it is possible that all hippocampal representations, even if distinct, under some conditions could be associated by chance with error trials (i.e., the encoding process was not "foolproof").

\section{Ensemble encoding uses "conjunctions" of task-relevant features}

A relatively small number of neurons (10) effectively encoded distinct behavioral events during the DNMS trial in the present study. However, ensemble encoding would not be expected to be more complex than the features of the DNMS task. Hence, because the task could be reduced to only four discrete behavioral events, large numbers of hippocampal neurons could have encoded similar (or even the same) information. Thus, reducing the number of events to be encoded, but still requiring those events to be retained across a delay interval (perhaps necessitating large numbers of neurons with similar codes), may have allowed this operational property to be revealed by recording from a relatively small to moderate number of distributed hippocampal neurons.

The above finding suggests that information content within the ensemble was maximized relative to the task contingency (Shannon, 1948; Hamming, 1986; Gochin et al., 1994). We have characterized information content in these ensembles under a number of different conditions of data acquisition (Hampson and Deadwyler, in press). The results of such analyses indicated that two of the most important aspects of assessing information content in ensembles of this size were as follows: (1) ability to detect multiple representations of task relevant events across different neurons in the ensemble, and (2) that the neurons encoding such information in the ensemble be recorded simultaneously. The latter feature is relevant because estimates of information content in ensembles of neurons derived or reconstructed from serially recorded single units across different times and behavioral sessions (Georgopoulos et al., 1989; Eskandar et al., 1992; Young and Yamane, 1992; Miller et al., 1993; Gochin et al., 1994; Schoenbaum and Eichenbaum, 1995) were considerably lower than we assessed from simultaneously recorded neurons (Hampson and Deadwyler, in press).

The identification of four distinct sources of variance (roots) within each of the seven ensembles of hippocampal neurons, coupled with the fact that those variance sources were associated with events in the behavioral classification scheme of the canonical analysis, is strong evidence for a specific type of neural representation in which more than one task-relevant feature is encoded by each neuron within the ensemble (Brown, 1982). Previous studies (Eichenbaum et al., 1987; Hampson et al., 1993b; Young et al., 1994) of single hippocampal neuron activity have demonstrated that a majority of hippocampal neurons encoded more than one feature of the task and that the dominant pattern of encoding was "conjunctive" in nature, combining represented features across more than one dimension (i.e., increased firing for left lever responses, but only during the Sample phase). This type of "conjunctive" encoding has been shown to exist in both cortical and hippocampal neurons (Brown, 1982; Rolls, 1991b). Such conjunctive encoding and multiple representation in single neurons within the ensemble would account for the relatively high information content in the small hippocampal ensembles recorded here, as hypothesized by Eichenbaum (1993).

In conclusion, the above findings give important insight into the types of hippocampal neural processes involved in spatial recognition memory as measured by this DNMS task. Several aspects of the findings support a single-process memory system with respect to distinctiveness of encoded information and its persistence across interposed delay intervals in tests of short-term memory. To our knowledge, this is one of the first demonstrations that ensemble encoding and retrieval of "functionally relevant" information are represented as distinct firing patterns in hippocampal networks. The data provide implications for several views of how hippocampal systems might perform such a function and give additional insight into how that process can be perturbed by either proactively or retroactively interfering factors. At this point, a more detailed analysis is needed to understand the dynamics of such processes with respect to mechanisms of pattcrn gencration within hippocampal ensembles. What has been demonstrated conclusively, however, is the utility and richness of knowledge obtainable from "many-neuron" ensemble recording techniques (Nicolelis et al., 1993a,b; Nicolelis and Chapin, 1994). Coupled with the appropriate application of multivariate statistical analyses to the large volume of neuron data generated, this approach has yielded insight into the mechanisms underlying how the brain sorts and represents information along multiple behavioral and cognitive dimensions.

\section{REFERENCES}

Aggleton JP, Keith AB, Rawlins JN, Hunt PR, Sahgal A (1992) Removal of the hippocampus and transection of the fornix produce comparable deficits on delayed non-matching to position by rats. Behav Brain Res 52:61-71.

Alvarez P, Zola-Morgan S, Squire LR (1995) Damage limited to the hippocampal region produces long-lasting memory impairment in monkeys. J Neurosci 15:3796-3807.

Amaral DG, Witter MP (1989) The three-dimensional organization of the hippocampal formation: a review of anatomical data. Neuroscience 31:571-591.

Amaral DG, Dolorfo C, Alvarez-Royo P (1991) Organization of CA1 projections to the subiculum: a PHA-L analysis in the rat. Hippocampus $1: 415-435$.

Angeli SJ, Murray EA, Mishkin M (1993) Hippocampectomized monkeys can remember one place but not two. Neuropsychologia 31:1021-1030.

Baylis GC, Rolls ET (1987) Responses of neurons in the inferior temporal cortex in short term and serial recognition memory tasks. Exp Brain Res 65:614-622.

Berger TW, Rinaldi PC, Weisz DJ, Thompson RF (1983) Single-unit analysis of different hippocampal cell types during classical conditioning of rabbit nictitating membranc responsc. J Neurophysiol 50:1197-1219.

Breese CR, Hampson RE, Deadwyler SA (1989) Hippocampal place cells: stereotypy and plasticity. J Neurosci 9:1097-1111.

Brown MW (1982) Effect of context on the responses of single units recorded from the hippocampal region of behaviourally trained monkeys. In: Neuronal plasticity and memory formation (Ajmone-Marsan C, Matthies H, eds). New York: Raven.

Christian EP, Deadwyler SA (1986) Behavioral functions and hippocampal cell types: evidence for two nonoverlapping populations in the rat. J Neurophysiol 55:331-348

Cohen NJ, Eichenbaum H (1993) Memory, amnesia, and the hippocampal system. Cambridge, MA: MIT.

Compton DM (1993) Encoding of a nonmonotonic serial pattern: role of the dorsal hippocampus and amygdala. Physiol Behav 53:657-665.

Deadwyler S $\Lambda$, Hampson RE (1995) Perspectives-ensemble activity and behavior: What's the code? Science, 470:1316-1318. 
Deadwyler SA, West M, Lynch G (1979) Activity of dentate granule cells during lcarning: differcntiation of perforant path input. Brain Res 169:29-43.

Dunnett SB (1985) Comparative effects of cholinergic drugs and lesions of nucleus basalis or fimbria-fornix on delayed matching in rats. Psychopharmacology 87:357-363.

Dunnett SB (1989) Comparison of short-term memory deficits in animal models of aging using an operant delayed response task in rats. In: The biology of memory (Squire LR, Lindenlaub E, eds), pp 581-603. New York: Schattauer Verlag.

Dunnett SB, Rogers DC, Jones GH (1989) Effects of nucleus basalis magnocellularis lesions in rats on delayed matching and non-matching to position tasks. Eur J Neurosci 1:395-406.

Eichenbaum H (1993) Thinking about brain assemblies. Science 261: 993-994.

Eichenbaum H, Kuperstein M, Fagan A, Nagode J (1987) Cue-sampling and goal-approach correlates of hippocampal unit activity in rats performing an odor discrimination task. J Neurosci 7:716-732.

Eichenbaum H, Otto T, Cohen NJ (1994) Two functional components of the hippocampal memory system. Behav Brain Sci 17:449-518.

Eskandar EN, Richmond BJ, Optican LM (1992) Role of inferior temporal neurons in visual memory. I. Temporal encoding of information about visual images, recalled images, and behavioral content. J Neurophysiol 68:1277-1295.

Funahashi S, Bruce CJ, Goldman-Rakic PS (1989) Mnemonic coding of visual space in the monkey's dorsolateral prefrontal cortex. J Neurophysiol 61:331-349.

Fuster JM, Jervey JP (1982) Neuronal firing in the inferotemporal cortex of the monkey in a visual memory task. J Neurosci $2: 361-375$.

Gaffan D (1993) Additive effects of forgetting and fornix transection in the temporal gradient of retrograde amnesia. Neuropsychologia 31:1055-1066.

Gaffan D, Murray EA (1992) Monkeys (Macaca fascicularis) with rhinal cortex ablations succeed in object discrimination learning despite $24 \mathrm{hr}$ intertrial intervals and fail at matching to sample despite double sample presentations. Behav Neurosci 106:30-38.

Gaffan D, Shields C, Harrison S (1984) Delayed matching by fornixtransected monkeys: the sample, the push and the bait. Q J Exp Psychol 36B:305-317.

Georgopoulos AP, Lurito JT, Petrides M, Schwartz AB, Massey JT (1989) Mental rotation of the neuronal population vector. Science 243:234-236.

Gluck MA, Myers CE (1995) Representation and association in memory: a neurocomputational view of hippocampal function. Curr Dir Psychol Sci 4:23-29.

Gochin PM, Colombo M, Dorfman GA, Gerstein GL, Gross CG (1994) Neural ensemble coding in inferior temporal cortex. J Neurophysiol 71:2325-2337.

Hamming RW (1986) Coding and information theory. Englewood Cliffs, NJ: Prentice-Hall.

Hampson RE, Deadwyler SA (1994) Hippocampal representations of DMS/DNMS in the rat. Behav Brain Sci 17:480-482.

Hampson RE, Bunn T, Byrd DR, Deadwyler SA (1993a) Modeling of spatial vs behavioral firing of hippocampal complex spike cells. Soc Neurosci Abstr 19:1608.

Hampson RE, Byrd DR, Deadwyler SA (1994) Population analyses reveal that rat hippocampus encodes both spatial and task-specific information during delayed-nonmatch-to-sample tasks. Soc Neurosci Abstr 20:430.

Hampson RE, Byrd DR, Konstantopoulos JK, Bunn T, Jarrard LE, Deadwyler SA (1995) Proactive interference and short-term memory during performance of a DNMS task in normal rats and rats with hippocampus removed. Soc Neurosci Abstr 21:1215.

Hampson RE, Deadwyler SA (1995) LTP and LTD and the encoding of memory in small ensembles of hippocampal neurons. In: Long-term potentiation, 3rd ed (Baudry M, Davis J, eds), in press.

Hampson RE, Heyser CJ, Deadwyler SA (1993b) Hippocampal cell firing correlates of delayed-match-to-sample performance in the rat. Behav Neurosci 107:715-739.

Hampson RE, Kirby MT, Alexander KE, King VC, Deadwyler SA (1992) Functional significance of anatomic connections between $\mathrm{CA} 1$ and $\mathrm{CA} 3$ hippocampal cells during delayed match to sample behavior in the rat. Soc Neurosci Abstr 18:1065.

Heyser CJ, Iampson RE, Deadwyler SA (1993) The effects of delta-9THC on delayed match to sample performance in rats: alterations in short-term memory produced by changes in task specific firing of hippocampal neurons. J Pharmacol Exp Ther 264:294-307.

Jarrard LE (1993) On the role of the hippocampus in learning and memory in the rat. Behav Neural Biol 60:9-26.

Jarrard LE, Davidson TL (1994) The hippocampus and complex, nonspatial discrimination: Is learning still "not possible"? In: Neurobehavioral plasticity: learning, development and response to brain insults (Spear NE, Spear LP, Woodruff M, eds).

Lawley DN (1959) Tests of significance in canonical analysis. Biometrika 46:59-66.

Markowska AL, Olton DS, Murray EA, Gaffan D (1989) A comparative analysis of the role of fornix and cingulate cortex in memory: rats. Exp Brain Res 74:187-201.

McNaughton BL, Morris RGM (1987) Hippocampal synaptic enhancement and information storage within a distributed memory system. Trends Neurosci 10:408-415.

McNaughton BL, O'Keefe J, Barnes CA (1983) The stereotrode: a new technique for simultaneous isolation of several single units in the central nervous system from multiple unit records. J Neurosci Methods 8:391-397.

Miller EK, Li L, Desimone R (1991) A neural mechanism for working and recognition memory in inferior temporal cortex. Science 254:1377-1379.

Miller EK, Li L, Desimone R (1993) Activity of neurons in anterior inferior temporal cortex during a short-term memory task. J Neurosci $13: 1460-1478$.

Miller R (1991) Cortico-hippocampal interplay and the representation of contexts in the brain. In: Studies of brain function (Braitenberg $V$, Barlow HB, Bullock TH, Florey E, Grusser O-J, Peters A, eds). New York: Springer.

Miyashita Y, Chang HS, Mori K (1992) A hypothesis on primal longterm memory: neurophysiological evidence in the primate temporal cortex. In: Neuropsychology of memory (Squire LR, Butters N, eds). New York: Guilford.

Murray EA, Davidson M, Gaffan D, Olton DS, Suomi S (1989) Effects of fornix transection and cingulate cortical ablation on spatial memory in rhesus monkeys. Exp Brain Res 74:173-186.

Nadel L (1991) The hippocampus and space revisited. Hippocampus $1: 221-229$.

Nicolelis MA, Chapin JK (1994) Spatiotemporal structure of somatosensory responses of many-neuron ensembles in the rat ventral posterior medial nucleus of the thalamus. J Neurosci 14:3511-3532.

Nicolelis MA, Lin RC, Woodward DJ, Chapin JK (1993a) Induction of immediate spatiotemporal changes in thalamic networks by peripheral block of ascending cutaneous information. Nature 361:533-536.

Nicolelis MA, Lin RC, Woodward DJ, Chapin JK (1993b) Dynamic and distributed properties of many-neuron ensembles in the ventral posterior medial thalamus of awake rats. Proc Natl Acad Sci USA 90:2212-2216.

O'Keefe JA, Nadel L (1978) The hippocampus as a cognitive map. Oxford: Clarendon.

Otto T, Eichenbaum H (1992) Complementary roles of the orbital prefrontal cortex and the perirhinal-entorhinal cortices in an odor-guided delayed-nonmatching-to-sample task. Behav Neurosci 106:762-775.

Peinado-Manzano MA (1990) The role of the amygdala and the hippocampus in working memory for spatial and non-spatial information. Behav Brain Res 38:117-134.

Rawlins JN, Lyford GL, Seferiades A, Deacon RM, Cassaday HJ (1993) Critical determinants of nonspatial working memory deficits in rats with conventional lesions of the hippocampus or fornix. Behav Neurosci 107:420-433.

Rawlins JNP, Maxwell TJ, Sinden JD (1988) The effects of fornix section on win-stay/lose-shift and win-shift/lose-stay performance in the rat. Behav Brain Res 31:17-28.

Rolls ET (1991a) Functions of the primate hippocampus in spatial and nonspatial memory. Hippocampus 1:258-261.

Rolls ET (1991b) The representation and storage of information in neuronal networks in the primate cerebral cortex and hippocampus. In: The computing neuron (Durbin R, Miall C, Mitchison G, eds), pp 125-159. New York: Addison-Wesley.

Rolls ET, Cahusac PM, Feigenbaum JD, Miyashita Y (1993) Responses of single neurons in the hippocampus of the macaque related to recognition memory. Exp Brain Res 93:299-306.

Rothblat LA, Kromer LF (1991) Object recognition memory in the rat: the role of the hippocampus. Behav Brain Res 42:25-32. 
Sakurai Y (1990a) Hippocampal cells have behavioral correlates during the performance of an auditory working memory task in the rat. Behav Neurosci 104:253-263.

Sakurai Y (1990b) Cells in the rat auditory system have behavioral correlates during the performance of an auditory working memory task in the rat. Behav Neurosci 104:856-868.

Schoenbaum G, Eichenbaum $\mathrm{H}$ (1995) Information coding in the rodent prefrontal cortex. II. Ensemble activity in orbitofrontal cortex. J Neurophysiol 74:751-762.

Shannon CE (1948) Bell System Technical Journal Monograph B-1598, v.27.

Squire LR (1992) Memory and the hippocampus: a synthesis from findings with rats, monkeys, and humans. Psychol Rev 99:195-231.

Stevens J (1992) Applied multivariate statistics for the social sciences. Hillsdale, NY: Lawrence Erlbaum.

Suzuki WA, Zola-Morgan S, Squire LR, Amaral DG (1993) Lesions of the perirhinal and parahippocampal cortices in the monkey produce long-lasting memory impairment in the visual and tactual modalities. J Neurosci 13:2430-2451.

Tonkiss J, Feldon J, Rawlins JN (1990) Section of the descending columns of the fornix produces delay- and interference-dependent working memory deficits. Behav Brain Res 36:113-126.

Volpe BT, Davis HP, Towle A, Dunlap WP (1992) Loss of hippocampal CA1 pyramidal neurons correlates with memory impairment in rats with ischemic or neurotoxin lesions. Behav Neurosci 106:457-464.

Watanabe T, Niki H (1985) Hippocampal unit activity and delayed response in the monkey. Brain Res 325:241-254.

Weiskrantz L (1991) Disconnected awareness for detecting, processing, and remembering in neurological patients. J R Soc Med 84:466-470.
Wiener SI, Paul CA, Eichenbaum H (1989) Spatial and behavioral correlates of hippocampal neuronal activity. J Neurosci 9:2737-2763.

Wilson FA, Riches IP, Brown MW (1990) Hippocampus and medial temporal cortex: neuronal activity related to behavioural responses during the performance of memory tasks by primates. Behav Brain Res 40:7-28.

Witter MP, Van Hoesen GW, Amaral DG (1989) Topographical organization of the entorhinal projection to the dentate gyrus of the monkey. J Neurosci 9:216-228.

Young BJ, Fox GD, Eichenbaum H (1994) Correlates of hippocampal complex-spike cell activity in rats performing a nonspatial radial mazc task. J Neurosci 14:6553-6563.

Young MP, Yamane S (1992) Sparse population coding of faces in the inferotemporal cortex. Science 256:1327-1331.

Zola-Morgan S, Squire L, Amaral DG (1989a) Lesions of the hippocampal formation but not lesions of the fornix or the mammillary nuclei produce long-lasting memory impairment in monkeys. J Neurosci 9:898-913.

Zola-Morgan S, Squire LR, Amaral DG (1989b) Lesions of the amygdala that spare adjacent cortical regions do not impair memory or exacerbate the impairment following lesions of the hippocampal formation. J Neurosci 9:1922-1936.

Zola-Morgan S, Squire LR, Amaral DG, Suzuki WA (1989c) Lesions of perirhinal and parahippocampal cortex that spare the amygdala and hippocampal formation produce severe memory impairment. J Neurosci 9:4355-4370.

Zola-Morgan S, Squire LR, Rempel NL, Clower RP, Amaral DG (1992) Enduring memory impairment in monkeys after ischemic damage to the hippocampus. J Neurosci 12:2582-2596. 